\title{
On Coprimality Graphs for Symmetric Groups
}

Ballantyne, John J and Greer, Nicholas M and Rowley, Peter J

2012

Manchester Institute for Mathematical Sciences

School of Mathematics

The University of Manchester

\footnotetext{
Reports available from: http://eprints.maths.manchester.ac.uk/

And by contacting: The MIMS Secretary

School of Mathematics

The University of Manchester

Manchester, M13 9PL, UK
} 


\title{
On Coprimality Graphs for Symmetric Groups
}

\author{
John Ballantyne, Nicholas Greer, Peter Rowley
}

\begin{abstract}
For $G$ a group, $X$ a subset of $G$ and $\pi$ a set of positive integers we define a graph $\mathcal{C}_{\pi}(G, X)$ whose vertex set is $X$ with $x, y \in X$ joined by an edge provided $x \neq y$ and the order of $x y$ is in $\pi$. Here we investigate $\mathcal{C}_{\pi}(G, X)$ when $G$ is a finite symmetric group and $X$ is a $G$-conjugacy class of elements of order $p, p$ a prime.
\end{abstract}

MSC 20B30

\section{Introduction}

Suppose that $G$ is a group, $X$ is a subset of $G$ and $\pi$ is a set of positive integers. We define the $\pi$-coprimality graph, $\mathcal{C}_{\pi}(G, X)$, to be the graph with vertex set $X$ where, for $x, y \in X, x$ and $y$ are adjacent in $\mathcal{C}_{\pi}(G, X)$ if $x \neq y$ and the order of $x y$ is in $\pi$. Because $x y$ and $y x$ are conjugate elements of $G$, we note that $\mathcal{C}_{\pi}(G, X)$ is an undirected graph. If the orders of the elements in $X$ are coprime to all the integers in $\pi$, we refer to $\mathcal{C}_{\pi}(G, X)$ as a $\pi$-coprimality graph (or just coprimality graph if $\pi$ is understood). We mention two important special cases of $\pi$-coprimality graphs, the first being when $X$ consists of involutions of $G$ and $\pi$ consists of all odd positive integers. Such graphs, called local fusion graphs and denoted by $\mathcal{F}(G, X)$, have been investigated by the authors in [2] when $G$ is a symmetric group and $X$ is a conjugacy class of involutions (see Theorem 2.2 in Section 2). While $\mathcal{C}_{\{2\}}(G, X)$ when $X$ is a $G$-conjugacy class of involutions is a commuting involution graph - such graphs have been studied in [3], [4], [5], [6] and [7]. Also certain types of coprimality graph appear in [9].

In this paper we investigate coprimality graphs for the finite symmetric groups and first address the question of connectedness. We shall use $p^{\prime}$ to denote the set of positive integers coprime to $p$.

Theorem 1.1. Suppose that $G=\operatorname{Sym}(n)$ and that $x$ is an element of order $p$, $p$ a prime. Let $X$ be the $G$-conjugacy class of $x$. Then $\mathcal{C}_{p^{\prime}}(G, X)$ is connected unless $n=4$ and $x$ has cycle type $2^{2}$.

We now turn to the question as to what can be said about the diameters of such graphs. For involution conjugacy classes we may give a complete answer (see [2]). Our results on conjugacy classes of elements of odd prime order are less complete. 
Theorem 1.2. Suppose that $G=\operatorname{Sym}(n)$ and $X$ is the $G$-conjugacy class of a p-cycle where $p$ is an odd prime. Then $\operatorname{Diam}\left(\mathcal{C}_{p^{\prime}}(G, X)\right)=2$ unless $n=3=p$ when $\operatorname{Diam}\left(\mathcal{C}_{p^{\prime}}(G, X)\right)=1$.

Theorem 1.3. Suppose that $G=\operatorname{Sym}(n)$ and $X$ is the $G$-conjugacy class of elements of cycle type $p^{r}$, where $p$ is an odd prime. If $r<\sqrt{p}$, then $\operatorname{Diam}\left(\mathcal{C}_{p^{\prime}}(G, X)\right) \leq 5$

Theorem 1.4. Suppose that $G=\operatorname{Sym}(n)$ and $X$ is the $G$-conjugacy class of elements of cycle type $p^{r}$, where $p \geq 5$ is prime. Let $k$ be the least non-negative integer such that $r / 2^{k} \leq\lfloor\sqrt{p}\rfloor$. Then $\operatorname{Diam}\left(\mathcal{C}_{p^{\prime}}(G, X)\right) \leq 5+k$.

This paper is arranged as follows. Section 2 develops some preliminary material much of which is deployed in the proof of Theorem 3.4. We also review certain results concerning the complex representation theory of the symmetric group, touching on theorems of Frame, Robinson and Thrall (Theorem 2.3) and the Murnaghan-Nakayama rule (Theorem 2.4). Section 3 begins with a number of results on the complex irreducible characters of the symmetric groups, our aim being to prove Lemma 3.3. With Lemma 3.3 to hand we then establish Theorem 3.4 which deals in particular with the important base case when $G=$ $\operatorname{Sym}(p)$ and $x$ is a $p$-cycle. In our build up to determining diameters (or bounds for diameters) we pause, in Proposition 3.5, to observe a formula for the number of vertices distance two from $x, x \in X$, when $p \geq 7$ and $G=\operatorname{Sym}(p)$. Then Proposition 3.6, with an easy calculation, determines the distance between $x$ and $y$ where $x, y \in X$ are disjoint $p$-cycles in $\operatorname{Sym}(n)$. Next we come to Lemma 3.7 which is pivotal in the proofs of Theorems 1.2, 1.3 and 1.4. After Lemma 3.7 we then present a proof of Theorem 1.2. Moving onto Theorems 1.3 and 1.4 we first introduce, in Definition 3.9, the important notion of disentangled and tangled pairs. Subsequently in Lemmas 3.10, 3.12, 3.13 and 3.14 such pairs of elements of $\operatorname{Sym}(n)$ are analyzed at length. In our final section we prove Theorem 1.1 the proof of this being delayed till then as Theorem 1.2 is required.

Our group theoretic notation is standard as found, for example, in [1].

Finally, we thank the referees for their helpful comments and suggestions.

\section{Background Results}

Throughout this paper $t$ will denote a fixed element of $\operatorname{Sym}(n)$, the symmetric group of degree $n$, with $t$ having order $p$, a prime. We will sometimes denote $\operatorname{Sym}(m)(m \in \mathbb{N})$ by $\operatorname{Sym}(\Omega)$ where $\Omega$ is an $m$-element set upon which the permutations act. For $g \in \operatorname{Sym}(\Omega)$, the support of $g$, $\operatorname{supp}(g)$, is $\Omega \backslash \operatorname{fix}(g)$, where fix $(g)=\left\{\alpha \in \Omega \mid \alpha^{g}=\alpha\right\}$. If $\alpha \in \Omega$, then $\mathcal{O}_{g}(\alpha)$ denotes the $\langle g\rangle$-orbit of $\alpha$. Alternatively, writing $g=g_{1} \ldots g_{r}$ as a product of pairwise disjoint cycles, $\mathcal{O}_{g}(\alpha)=\operatorname{supp}\left(g_{j}\right)$ where $\alpha$ appears in the cycle $g_{j}$.

For a graph $\Gamma$ we use $d_{\Gamma}($,$) , or just d($, ) if there is no danger of confusion, to denote the standard graph theoretic distance on $\Gamma$. We recall the diameter of $\Gamma$, denoted $\operatorname{Diam}(\Gamma)$, is $\max \{d(x, y) \mid x, y \in V(\Gamma)\}, V(\Gamma)$ being the vertex set 
of $\Gamma$. Also for $i \in \mathbb{N} \cup\{0\}$ and $x \in V(\Gamma)$, the $i^{t h}$ disc of $x, \Delta_{i}(x)$, is defined to be

$$
\Delta_{i}(x)=\{y \in V(\Gamma) \mid d(x, y)=i\} .
$$

So $\Delta_{0}(x)=\{x\}$ and $\Delta_{1}(x)$ consists of the neighbours in $\Gamma$ of $x$.

Lemma 2.1. Let $\Gamma$ be a regular graph with $V(\Gamma)=X$. Let $x \in X$. If $\left|\Delta_{1}(x)\right|>$ $|X| / 2$, then $\Gamma$ is connected and $\operatorname{Diam}(\Gamma) \leq 2$.

Proof. Since $\left|\Delta_{1}(x)\right|>|X| / 2$, the regularity of $\Gamma$ implies connectedness. Suppose there exists $y \in X$ such that $d(x, y)=3$. Then $\Delta_{1}(x) \cap \Delta_{1}(y)=\emptyset$, since otherwise $d(x, y) \leq 2$. Therefore

$$
\left|\Delta_{1}(x)\right| \leq|X|-\left|\Delta_{1}(y)\right|=|X|-\left|\Delta_{1}(x)\right|
$$

by regularity. Hence $\left|\Delta_{1}(x)\right| \leq|X| / 2$, a contradiction. Thus the diameter of $\Gamma$ is at most 2 .

Theorem 2.2. Suppose that $G=\operatorname{Sym}(n)$ with $n \geq 5$ and $X$ is a $G$-conjugacy class of involutions. Then $\operatorname{Diam}(\mathcal{F}(G, X))=2$.

Proof. See [2].

The following results will be called upon in Section 3. Let $G$ be a finite group, with conjugacy classes $\mathcal{K}_{1}, \ldots, \mathcal{K}_{l}$, and let $K_{1}, \ldots, K_{l}$ be the corresponding class sums in the group algebra $\mathbb{C} G$. Let $a_{i j k}$ be the integers defined by

$$
K_{i} K_{j}=\sum_{k=1}^{l} a_{i j k} K_{k} .
$$

These integers are known as the class structure constants. Note that $a_{i j k}$ is precisely the number of pairs of elements $(x, y)$, where $x \in \mathcal{K}_{i}, y \in \mathcal{K}_{j}$, such that $x y=z$, where $z$ is some fixed element of $\mathcal{K}_{k}$. Now let $\left\{g_{1}, \ldots, g_{l}\right\}$ be a complete set of conjugacy class representatives for $G$. Then we have

$$
a_{i j k}=\frac{\left|\mathcal{K}_{i}\right|\left|\mathcal{K}_{j}\right|}{|G|} \sum_{\chi \in \operatorname{Irr}(G)} \frac{\chi\left(g_{i}\right) \chi\left(g_{j}\right) \overline{\chi\left(g_{k}\right)}}{\chi(1)} .
$$

The integers $a_{i j k}$ are therefore determined by the character table of $G$.

Suppose now that $G=\operatorname{Sym}(n)$, where $n \geq 5$, and let $X$ be the conjugacy class of $t$ in $G$. Furthermore, suppose that $p=n, n-1$ or $n-2$. We now consider the graph $\mathcal{C}_{p^{\prime}}(G, X)$. From our assumption on $n$, if $x \in X$ and $x \neq t$, then $x$ lies outside $\Delta_{1}(t)$ if and only if $o(t x)=p$ (where $o(t x)$ denotes the order of $t x$ ), so if and only if $t x \in X$ (note that $t x$ must be an even permutation, so cannot be a (disjoint) product of a $p$-cycle and a transposition). If we can count the number of such elements, and show that it is not greater than $|X| / 2$, then by Lemma $2.1 \mathcal{C}_{p^{\prime}}(G, X)$ is connected, and $\operatorname{Diam}\left(\mathcal{C}_{p^{\prime}}(G, X)\right) \leq 2$. By applying 
the above formula for the class structure constants, we have an expression for this number, namely

$$
\begin{aligned}
\left|X-\Delta_{1}(t)\right| & =\frac{|X|^{2}}{|G|} \sum_{\chi \in \operatorname{Irr}(G)} \chi(t) \chi(t) \overline{\chi(t)} / \chi(1) \\
& =\frac{|G|}{\left|C_{G}(t)\right|^{2}} \sum_{\chi \in \operatorname{Irr}(G)} \frac{\chi(t)}{\chi(1)}|\chi(t)|^{2} .
\end{aligned}
$$

The study of this case therefore reduces to the study of the character table of the symmetric group. Fortunately, an extensive theory exists on this topic. We briefly summarise the results which we require, and for a detailed treatment refer the reader to [10].

Let $n \in \mathbb{N}$. A partition of $n$ is a sequence

$$
\lambda=\left(\lambda_{1}, \lambda_{2}, \ldots, \lambda_{k}\right)
$$

where the $\lambda_{i}$ are weakly decreasing and $\sum_{i=1}^{k} \lambda_{i}=n$. Recall that the partitions of $n$ are in one to one correspondence with the complex irreducible representations of the symmetric group $\operatorname{Sym}(n)$. To each partition $\lambda$ we associate a Young diagram $D_{\lambda}$, consisting of $n$ cells and $k$ rows, left justified, with the length of row $i$ equal to $\lambda_{i}$. For example, for the partition $(5,4,4,2,1)$ of 16 , the corresponding Young diagram is shown below.

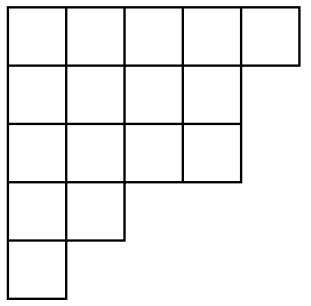

If $(i, j)$ is a cell in the diagram $D_{\lambda}$ of a partition $\lambda$, the hook $H_{i, j}$ is defined as

$$
H_{i, j}=\left\{\left(i, j^{\prime}\right) \mid j^{\prime} \geq j\right\} \cup\left\{\left(i^{\prime}, j\right) \mid i^{\prime} \geq i\right\} .
$$

We define the corresponding hook length as $h_{i, j}=\left|H_{i, j}\right|$. To illustrate, for our previous example, the hook $H_{1,1}$ is shaded, which has hook length $h_{1,1}=9$.

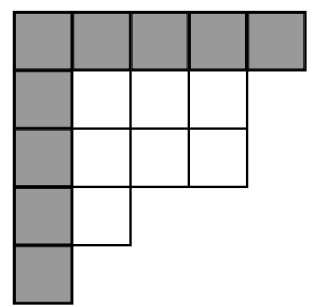

We may now state the first of our required results, known as the 'hook formula'. 
Theorem 2.3 (Frame, Robinson, Thrall). Let $\lambda$ be a partition of a natural number $n$, with $\chi^{\lambda}$ the character afforded by the corresponding irreducible representation of $\operatorname{Sym}(n)$. Then

$$
\chi^{\lambda}(1)=\frac{n !}{\prod_{(i, j) \in D_{\lambda}} h_{i, j}} .
$$

Again, we illustrate using our previous example. The length of each hook is displayed below.

\begin{tabular}{|c|c|c|c|c|}
\hline 9 & 7 & 5 & 4 & 1 \\
\hline 7 & 5 & 3 & 2 & \\
\hline 6 & 4 & 2 & 1 & \\
\hline 3 & 1 & & & \\
\hline 1 & & & & \\
\hline
\end{tabular}

Now, the hook formula yields

$$
\chi^{\lambda}(1)=\frac{16 !}{9 \cdot 7^{2} \cdot 6 \cdot 5^{2} \cdot 4^{2} \cdot 3^{2} \cdot 2^{2} \cdot 1^{4}}=549120 .
$$

Let $H_{i, j}$ be a hook in the Young diagram associated with some partition $\lambda$. Then the rim hook $R_{i, j}$ is obtained by projecting $H_{i, j}$ along diagonals onto the lower-right boundary of our diagram. Note that $\left|R_{i, j}\right|=\left|H_{i, j}\right|=h_{i, j}$. The leg length of $R_{i, j}$ is defined as

$$
l l\left(R_{i, j}\right)=\left(\text { number of rows of } R_{i, j}\right)-1
$$

For our example, the rim hook $R_{1,1}$ is shown, for which $l l\left(R_{1,1}\right)=4$.

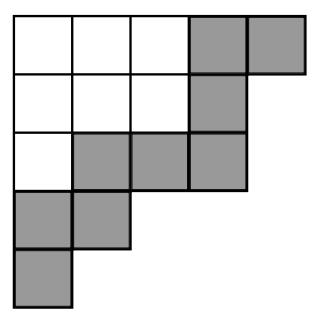

Observe that if $h_{i, j}<n$, and we remove the rim hook $R_{i, j}$ from the diagram $D_{\lambda}$, what remains is a Young diagram associated with some partition of $n-h_{i, j}$. We denote this new diagram by $D_{\lambda} \backslash R_{i, j}$.

For a conjugacy class $\mathcal{K}$ of $\operatorname{Sym}(n)$, we can naturally associate a partition of $n$ with $\mathcal{K}$ via the cycle type of elements of $\mathcal{K}$. We are now in a position to state the second of our required results, a combinatorial rule for calculating the values of irreducible characters of the symmetric group. 


\section{The Murnaghan-Nakayama Rule}

Let $\lambda$ be a partition of $n$, with corresponding irreducible character $\chi^{\lambda}$ of $\operatorname{Sym}(n)$.

Let $\sigma \in \operatorname{Sym}(n)$ have cycle type with associated partition $\mu=\left(\mu_{1}, \mu_{2}, \ldots, \mu_{r}\right)$

of $n$. We generate a branch $B$, and a corresponding value $c_{B} \in\{-1,0,1\}$, by using the following iterative procedure.

Initially, set $c_{0}=1$ and $D_{\lambda_{0}}=D_{\lambda}$. For $k \geq 1$, the $k$-th step is as follows:

1. If $D_{\lambda_{k-1}}$ consists of zero cells, set $c_{B}=c_{k-1}$, and stop.

2. If possible, remove a rim hook $R_{k}$ of length $\mu_{k}$ from $D_{\lambda_{k-1}}$, such that $D_{\lambda_{k-1}} \backslash R_{k}$ is a Young diagram, or consists of zero cells. If this is not possible, set $c_{B}=0$, and stop.

3. Set $c_{k}=(-1)^{l l\left(R_{k}\right)} \cdot c_{k-1}$, and $D_{\lambda_{k}}=D_{\lambda_{k-1}} \backslash R_{k}$.

When applying this rule, at each step, different choices of rim hook removal yield distinct branches. The totality of these branches (those generated by all possible valid combinations of rim hook removals) can be considered to form a tree $T$ associated with the pair $(\lambda, \mu)$. We have the following result.

Theorem 2.4 (Murnaghan-Nakayama). With the set-up as above, we have

$$
\chi^{\lambda}(\sigma)=\sum_{B} c_{B}
$$

where the sum runs over all distinct branches $B$ of $T$.

We illustrate the Murnaghan-Nakayama rule with an example. Let $n=10$, with partition $\lambda=(5,3,1,1)$, and let $\sigma \in \operatorname{Sym}(n)$ have cycle type 5.2.2.1. The tree we generate is as follows: 


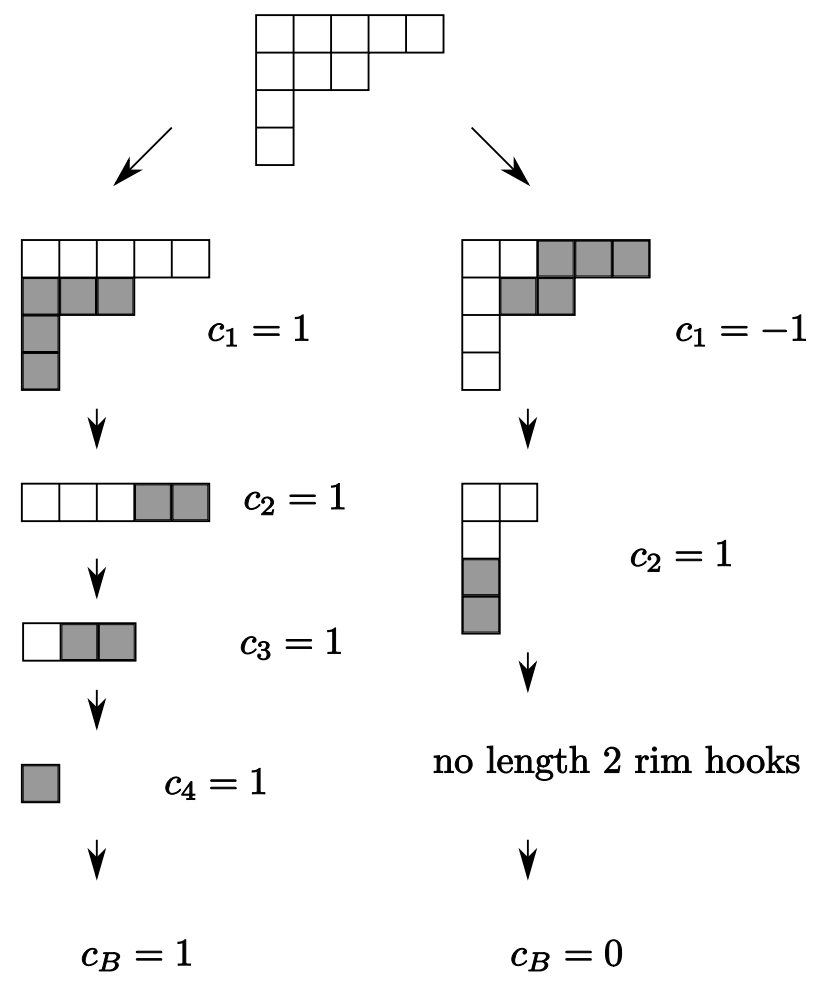

So for this example we have two branches to sum over, yielding $\chi^{\lambda}(\sigma)=1+0=$ 1.

\section{The Diameter of $\mathcal{C}_{p^{\prime}}(G, X), p$ odd}

In this section we prove Theorems 1.2, 1.3 and 1.4. We begin with some preparatory lemmas.

Lemma 3.1. Let $T$ be a Young diagram consisting of $n$ cells, and let $k>n / 2$. Then there is at most one way of removing a rim hook of length $k$ from $T$.

Proof. Suppose we have a way of removing a rim hook $R_{1}$ of length $k$ from $T$. After removal, a diagram of $n-k$ cells remains, which we denote $T_{1}$. Let $r$ and $r_{1}$ be the lengths of the top rows of $T$ and $T_{1}$ respectively. Similarly, denote by $c$ and $c_{1}$ the lengths of the first columns. 


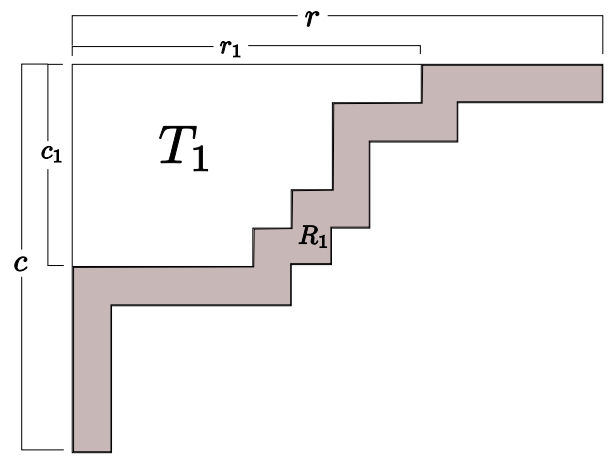

Note that since $k>n / 2$, either $r>r_{1}, c>c_{1}$, or both. Firstly, suppose both hold. Then clearly $R_{1}$ is maximal in the sense that any other rim hook which can be removed from $T$ is of length less than $k$. Thus $R_{1}$ is our only choice.

Suppose now that either $r=r_{1}$ or $c=c_{1}$. Since if necessary we may just reflect the diagram in the main diagonal, without loss of generality we may assume $c=c_{1}$. Let $H_{1, j}$ be the hook from which $R_{1}$ is projected, based at position $(1, j)$ in $T$. Suppose there exists another possible choice of rim hook, labelled $R_{2}$, with corresponding diagram $T_{2}$.

Any projection of a hook $H_{1, j-d}$, where $d \geq 1$, will have length greater than $k$, so is not suitable. Also, a projection of a hook $H_{i, j}$, where $i>1$ and $j>1$, will have length less than $n / 2<k$, again unsuitable. Thus $R_{2}$ must project from some position $(i, 1)$ where $i>1$.

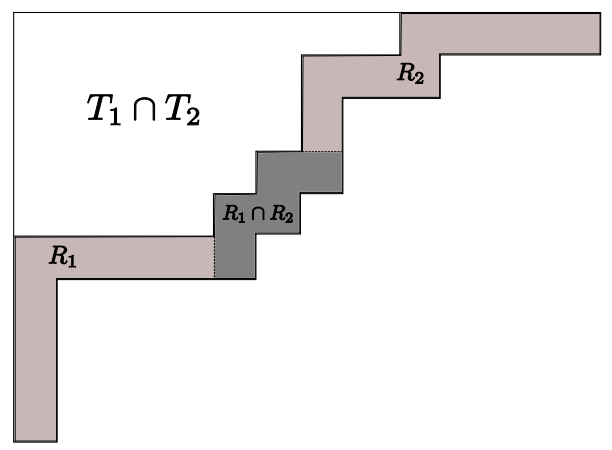

Note that $R_{1}$ and $R_{2}$ must intersect nontrivially, since otherwise

$$
|T| \geq\left|R_{1}\right|+\left|R_{2}\right|=2 k>n,
$$

a contradiction. Since $R_{1}$ projects from $(1, j)$, and $R_{2}$ projects from $(i, 1)$, $R_{1} \cap R_{2}$ must intersect in the empty set with both the first row and first column of $T$. Consequently we have $\left|T_{1} \cap T_{2}\right| \geq\left|R_{1} \cap R_{2}\right|$. Thus

$$
|T|=\left|R_{1} \cup R_{2}\right|+\left|T_{1} \cap T_{2}\right| \geq\left|R_{1} \cup R_{2}\right|+\left|R_{1} \cap R_{2}\right|=2 k>n,
$$

another contradiction. Therefore $R_{1}$ is the only suitable choice of rim hook. 
Lemma 3.2. Let $\chi_{S}$ be the complex character of $\operatorname{Sym}(n)$ associated to a diagram $S$, and let $\chi_{T}$ be the complex character of $\operatorname{Sym}(n-k)$ associated to the diagram $T$, where $T$ is obtained by removing a rim hook of length $k$ from $S$, where $k>n / 2$. Assume $\chi_{S}$ and $\chi_{T}$ are both nonlinear. Then $\chi_{S}(1) \geq 2 \chi_{T}(1)$.

Proof. We use the Murnaghan-Nakayama rule to calculate $\chi_{S}(1)$. Denote by $R$ the rim hook which we remove from $S$ to obtain $T$.

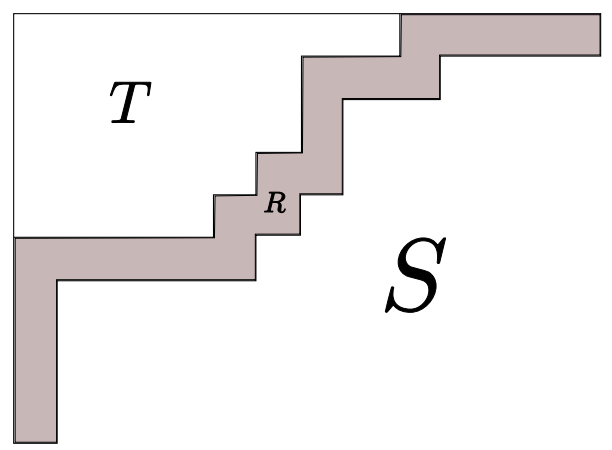

Denote by $r_{T}$ and $r_{S}$ the lengths of the top rows of $T$ and $S$ repectively. Similarly denote by $c_{T}$ and $c_{S}$ the lengths of the first columns. As $k>n / 2$, note that either $r_{S}>r_{T}, c_{S}>c_{T}$, or both. The first step in calculating $\chi_{S}(1)$ is to remove a single cell rim hook from $S$. Clearly it is possible to remove this cell from $R$.

We now consider the following three cases: either $r_{S} \neq r_{T}+1$ or $c_{S} \neq c_{T}+1$ and $R$ is not a single row (or column); both $r_{S}=r_{T}+1$ and $c_{S}=c_{T}+1$; or $R$ consists of only a single row (or column). First, suppose that either $r_{S} \neq r_{T}+1$ or $c_{S} \neq c_{T}+1$, and that $R$ does not consist only of a single row (or column). Then we have at least two choices of single cell rim hook removal from $R$. For each choice, we are able to continue to remove single cells from $R$ until the diagram $T$ remains. The value we calculate from this point onwards is $\chi_{T}(1)$. But now, the details of the Murnaghan-Nakayama rule yield $\chi_{S}(1) \geq 2 \chi_{T}(1)$.

Suppose now that $r_{S}=r_{T}+1$ and $c_{S}=c_{T}+1$ (this situation can only occur when $n \leq 9)$.

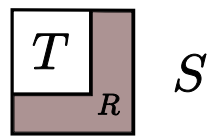

It is clear from the figure that after our first removal we have two choices of single cell removal. Arguing as above, we again have $\chi_{S}(1) \geq 2 \chi_{T}(1)$.

Finally, suppose that $R$ consists of only a single row (or column). Note that since $\chi_{T}$ and $\chi_{S}$ are both non-linear, $T$ must have at least two rows (or columns). 


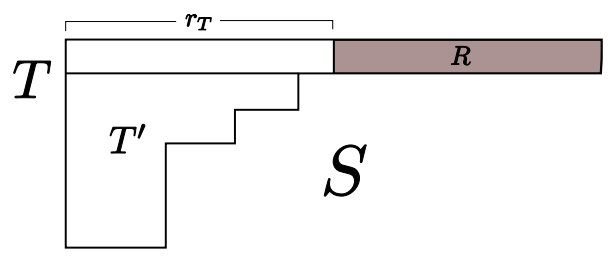

In this case we use the hook formula, which yields

$$
\chi_{T}(1)=\frac{(n-k) !}{\prod_{(i, j) \in T} h_{i, j}^{T}}
$$

and

$$
\chi_{S}(1)=\frac{n !}{\prod_{(i, j) \in S} h_{i, j}^{S}},
$$

where $h_{i, j}^{T}$ denotes the length of the hook based at $(i, j)$ in the diagram $T$, and $h_{i, j}^{S}$ the length of the hook based at $(i, j)$ in the diagram $S$. By observing the position of the subdiagram $T^{\prime}$ in both $T$ and $S$, we see that

$$
\chi_{T}(1)=\frac{(n-k)(n-k-1) \cdots\left(\left|T^{\prime}\right|+1\right)}{\prod_{(i, j) \in T \backslash T^{\prime}} h_{i, j}^{T}} \cdot B
$$

and

$$
\chi_{S}(1)=\frac{n(n-1) \cdots\left(\left|T^{\prime}\right|+1\right)}{\prod_{(i, j) \in S \backslash T^{\prime}} h_{i, j}^{S}} \cdot B,
$$

where

$$
B=\frac{\left|T^{\prime}\right| !}{\prod_{(i, j) \in T^{\prime}} h_{i, j}^{T^{\prime}}}
$$

We must therefore show that

$$
\frac{n(n-1) \cdots\left(\left|T^{\prime}\right|+1\right)}{\prod_{(i, j) \in S \backslash T^{\prime}} h_{i, j}^{S}} \geq \frac{2 \cdot(n-k)(n-k-1) \cdots\left(\left|T^{\prime}\right|+1\right)}{\prod_{(i, j) \in T \backslash T^{\prime}} h_{i, j}^{T}} .
$$

Note that as $k>n / 2$ and $\chi_{T}$ is non-linear, $r_{T}<k-1$. Hence we have

$$
\begin{aligned}
h_{1, r_{T}}^{T} & \geq h_{1, r_{T}+k}^{S}, \\
h_{1, r_{T}-1}^{T} & \geq h_{1, r_{T}+k-1}^{S}, \\
& \vdots \\
h_{1,1}^{T} & \geq h_{1, k+1}^{S} .
\end{aligned}
$$


Also

$$
\begin{aligned}
h_{1,1}^{S} & \leq n, \\
h_{1,2}^{S} & \leq n-2, \quad \text { (as } \chi_{T} \text { is non-linear) } \\
h_{1,3}^{S} & \leq n-3, \\
h_{1,4}^{S} & \leq n-4, \\
& \vdots \\
h_{1, k-1}^{S} & \leq n-k+1 .
\end{aligned}
$$

Finally, note that $h_{1, k}^{S}=r_{T}+1 \leq(n-1) / 2$. Indeed,

$$
n-1 \geq r_{T}+k>r_{T}+r_{T}+1=2 r_{T}+1,
$$

so $n-1 \geq 2 r_{T}+2$. Hence we have

$$
\frac{n(n-1) \cdots\left(\left|T^{\prime}\right|+1\right)}{\prod_{(i, j) \in S \backslash T^{\prime}} h_{i, j}^{S}} \geq \frac{2 \cdot(n-k)(n-k-1) \cdots\left(\left|T^{\prime}\right|+1\right)}{\prod_{(i, j) \in T \backslash T^{\prime}} h_{i, j}^{T}},
$$

as required.

Lemma 3.3. Let $G=\operatorname{Sym}(n)$, and let $X$ be a conjugacy class of $k$-cycles in $G$, where $k>n / 2$. Then for a non-linear complex irreducible character $\chi$ of $G$ we have

$$
|\chi(x)| \leq \chi(1) / 2
$$

for all $x \in X$.

Proof. By the Murnaghan-Nakayama rule, the first step in calculating $\chi(x)$ is to remove a rim hook of length $k$ (if possible) from the diagram $T$ associated with $\chi$. If this is not possible, then $\chi(x)=0$, and the result clearly holds. Therefore suppose it is possible. Then by Lemma 3.1, there is only one way to do this.

If $k=n$, then clearly $\chi(x)= \pm 1$, and the result follows since $\chi$ is nonlinear. So now suppose $k<n$. After removing our rim hook of length $k$, the Murnaghan-Nakayama rule tells us to remove single cell rim hooks from the remaining diagram $T^{\prime}$ in all possible ways. However, the hook formula also yields this value. Thus

$$
\chi(x)= \pm \frac{(n-k) !}{\prod_{(i, j) \in T^{\prime}} h_{i, j}^{T^{\prime}}}= \pm \chi_{T^{\prime}}(1)
$$

where $\chi_{T^{\prime}}$ is the character of $\operatorname{Sym}(n-k)$ associated with $T^{\prime}$. But $T^{\prime}$ was obtained from $T$ by removing a rim hook of length $k>n / 2$. Hence Lemma 3.2 implies $\chi(1) \geq 2 \chi_{T^{\prime}}(1)$. Thus $|\chi(x)| \leq \chi(1) / 2$.

We are now in a position to apply our results to obtain information about our graphs in the cases when $p=n, n-1$ and $n-2$. 
Theorem 3.4. Let $G=\operatorname{Sym}(n)$, where $n \geq 5$, and let $X$ be a conjugacy class of $p$-cycles in $G$, where $p=n, n-1$ or $n-2$. Then $\mathcal{C}_{p^{\prime}}(G, X)$ is connected and $\operatorname{Diam}\left(\mathcal{C}_{p^{\prime}}(G, X)\right)=2$.

Proof. When $n<15$ we can check directly using MAGMA [8], so suppose $n \geq 15$. Let $t=(1,2, \ldots, p) \in X$. As observed in Section 2, since $p=n, n-1$ or $n-2$, $x \in X$ lies outside $\Delta_{1}(t)$ if and only if $o(t x)=p$, so if and only if $t x \in X$. The class structure constants yield the following:

$$
\begin{aligned}
\left|X-\Delta_{1}(t)\right| & =\frac{|X|^{2}}{|G|} \sum_{\chi \in \operatorname{Irr}(G)} \chi(t) \chi(t) \overline{\chi(t)} / \chi(1) \\
& =\frac{|G|}{\left|C_{G}(t)\right|^{2}} \sum_{\chi \in \operatorname{Irr}(G)} \frac{\chi(t)}{\chi(1)}|\chi(t)|^{2} .
\end{aligned}
$$

Since $G^{\prime} \cong \operatorname{Alt}(n)$, we have $\left[G: G^{\prime}\right]=2$, so $G$ has exactly two linear characters. Also, since $p$ is odd, $\chi(t)=1$ for both of these characters. Denote by $\operatorname{Irr}(G)^{*}$ the set of non-linear irreducible characters of $G$. Then

$$
\sum_{\chi \in \operatorname{Irr}(G)} \frac{\chi(t)}{\chi(1)}|\chi(t)|^{2}=2+\sum_{\chi \in \operatorname{Irr}(G)^{*}} \frac{\chi(t)}{\chi(1)}|\chi(t)|^{2} .
$$

By Lemma 3.3, for non-linear $\chi \in \operatorname{Irr}(G)$ we have $|\chi(t)| / \chi(1) \leq 1 / 2$. Furthermore, as $n \geq 15$ we have $p \geq 13$, and there are at least 11 non-zero character values on $X$, and so at least 4 negative character values on $X$ (this can be easily verified by considering possible Young diagrams and using the MurnaghanNakayama rule). Hence we may write

$$
\begin{aligned}
\sum_{\chi \in \operatorname{Irr}(G)} \frac{\chi(t)}{\chi(1)}|\chi(t)|^{2} & \leq 2+\frac{1}{2}\left(\sum_{\chi \in \operatorname{Irr}(G)^{*}}|\chi(t)|^{2}-4\right) \\
& =\frac{1}{2} \sum_{\chi \in \operatorname{Irr}(G)^{*}}|\chi(t)|^{2} .
\end{aligned}
$$

Next, we apply column orthogonality, remembering that $G$ has exactly two linear characters.

$$
\begin{aligned}
\frac{1}{2} \sum_{\chi \in \operatorname{Irr}(G)^{*}}|\chi(t)|^{2} & =\frac{1}{2}\left(\left|C_{G}(t)\right|-2\right) \\
& =\frac{\left|C_{G}(t)\right|}{2}-1 \\
& <\frac{\left|C_{G}(t)\right|}{2} .
\end{aligned}
$$

Therefore

$$
\left|X-\Delta_{1}(t)\right|<\frac{|G|}{\left|C_{G}(t)\right|^{2}} \cdot \frac{\left|C_{G}(t)\right|}{2}=\frac{|X|}{2} .
$$

By Lemma 2.1, the result follows. 
In certain cases we can go further, and obtain an exact expression for the size of the second disc.

Proposition 3.5. Let $G=\operatorname{Sym}(p)$, where $p$ is a prime, $p \geq 7$. Write $p=$ $2 m+1$. Let $X$ be the conjugacy class of $p$-cycles, with $t=(1,2, \ldots, p) \in X$. Then the size of $\Delta_{2}(t)$ in the graph $\mathcal{C}_{p^{\prime}}(G, X)$ is given by the following:

$$
\left|\Delta_{2}(t)\right|=\frac{2 D}{p}-1
$$

where

$$
\begin{array}{r}
D=(p-1) !-(p-2) !+(-1)^{2} 2 !(p-3) !+(-1)^{3} 3 !(p-4) !+\cdots \\
\cdots+(-1)^{m-1}(m-1) !(p-m) !+(-1)^{m} \frac{1}{2} m !(p-m-1) ! .
\end{array}
$$

Proof. From Theorem 3.4 we have that $\mathcal{C}_{p^{\prime}}(G, X)$ is connected and has diameter 2 , and that

$$
\left|\Delta_{2}(t)\right|+1=\frac{|G|}{\left|C_{G}(t)\right|^{2}} \sum_{\chi \in \operatorname{Irr}(G)} \frac{\chi(t)}{\chi(1)}|\chi(t)|^{2} .
$$

Furthermore, after a moment's consideration we deduce that $\chi(t)= \pm 1$ for exactly $p$ irreducible characters (the 'L-shaped' diagrams, so those consisting of at most one row and one column), with the remainder yielding 0 on $X$. We use the hook formula to calculate $\chi(1)$ for each of these contributing characters.

Starting from the diagram of one single row, and adding one cell to the first column (and removing one cell from the top row) each time, the hook formula yields the following character degrees:

$$
1, p-1, \frac{(p-1)(p-2)}{2}, \frac{(p-1)(p-2)(p-3)}{3 \cdot 2}, \ldots, \frac{(p-1) \cdots(p-r)}{r !}, \ldots,
$$

where the sequence is symmetric around the $(m+1)$-th term. We also note that diagrams with an odd number of rows yield a character value of 1 , while those with an even number of rows yield -1 . Putting all this information together, along with the observations that $|G|=p$ ! and $\left|C_{G}(t)\right|=p$, gives us the desired expression for $\left|\Delta_{2}(t)\right|$.

Proposition 3.6. Suppose $G=\operatorname{Sym}(n)$, and $t$ is a p-cycle where $p \geq 3$ is prime. Let $X=t^{G}$. If $x \in X$ is disjoint from $t$, then there exists $y \in X$ with $d(t, y)=d(y, x)=1$.

Proof. Without loss of generality we assume

$$
t=(1,2, \ldots, p)
$$

and

$$
x=(p+1, p+2, \ldots, 2 p) .
$$


Take

$$
y=(1,2, \ldots, p-2, p+1, p+2) \in X .
$$

Then

$$
t y=(1,3,5, \ldots, p-2, p-1, p, 2,4,6, \ldots, p-3, p+1, p+2)
$$

and

$$
y x=(1,2, \ldots, p-2, p+2)(p-1)(p)(p+1, p+3, p+4, \ldots, 2 p) .
$$

Hence $t y$ has order $p+2$ and $y x$ has order $p-1$, so proving the result.

Suppose $x \in \operatorname{Sym}(\Omega)$, and that $\alpha \in \Omega$. We recall that $\mathcal{O}_{x}(\alpha)$ denotes the $\langle x\rangle$ orbit which contains $\alpha$. The following lemma is the key to proving Theorems 1.2, 1.3 and 1.4 .

Lemma 3.7. Let $G=\operatorname{Sym}(n)$, with $x, y \in G$ distinct elements of order at least 3. Denote by $\Omega$ the set upon which $G$ acts naturally. Suppose there exist distinct $\alpha, \beta, \gamma \in \Omega$ such that $\beta, \gamma \in \mathcal{O}_{y}(\alpha)$ but $\beta, \gamma \notin \mathcal{O}_{x y}(\alpha)$.

(i) If $\gamma \notin \mathcal{O}_{x y}(\beta)$, then there exists $z \in G$, where $\langle z\rangle$ has the same orbits on $\Omega$ as $\langle y\rangle$, such that $\left|\mathcal{O}_{x z}(\alpha)\right|=\left|\mathcal{O}_{x y}(\alpha)\right|+\left|\mathcal{O}_{x y}(\beta)\right|+\left|\mathcal{O}_{x y}(\gamma)\right|$.

(ii) If $\gamma \in \mathcal{O}_{x y}(\beta)$, then there exists $z \in G$, where $\langle z\rangle$ has the same orbits on $\Omega$ as $\langle y\rangle$, such that $\left|\mathcal{O}_{x z}(\alpha)\right|=\left|\mathcal{O}_{x y}(\alpha)\right|+c$ and $\left|\mathcal{O}_{x z}(\gamma)\right|=\left|\mathcal{O}_{x y}(\gamma)\right|-c$, where $c \geq 1$.

(iii) If $\left|\mathcal{O}_{y}(\alpha) \cap\left(\mathcal{O}_{x y}(\alpha) \cup \mathcal{O}_{x y}(\beta)\right)\right|=m$, where $m \geq 4$, then there exist

$$
M=(m-1)(m-2) / 2
$$

distinct elements $z_{1}, \ldots, z_{M}$, which can be created by an application of (ii), where for $1 \leq i \leq M$ each $\left\langle z_{i}\right\rangle$ has the same orbits on $\Omega$ as $\langle y\rangle$. Moreover, there exist natural numbers $c_{1}<c_{2}<\cdots<c_{m-2}$, along with $c_{0}:=0$, such that

$$
\left\{\left|\mathcal{O}_{x z_{k}}(\alpha)\right| \mid 1 \leq k \leq M\right\}=\left\{c_{i}-c_{j} \mid 0 \leq j<i \leq m-2\right\},
$$

and this set has cardinality at least $m-2$.

Proof. Without loss of generality we may suppose $y$ contains the cycle

$$
\sigma=\left(\delta_{1}, \alpha, \delta_{3}, \ldots, \delta_{k}, \beta, \delta_{k+2}, \ldots, \delta_{l}, \gamma, \delta_{l+2}, \ldots\right) .
$$

Firstly, suppose that $\beta$ and $\gamma$ lie in separate orbits of $\langle x y\rangle$. Then $x y$ contains the following cycles:

$$
\left(\delta_{1}^{x^{-1}}, \alpha, \ldots\right)\left(\delta_{k}^{x^{-1}}, \beta, \ldots\right)\left(\delta_{l}^{x^{-1}}, \gamma, \ldots\right),
$$


where $\delta_{1}^{x^{-1}}$ denotes the inverse image of $\delta_{1}$ under $x, \delta_{k}^{x^{-1}}$ the inverse image of $\delta_{k}$ under $x$ and $\delta_{l}^{x^{-1}}$ the inverse image of $\delta_{l}$ under $x$. Now let

$$
\bar{\sigma}=\left(\delta_{1}, \beta, \delta_{k+2}, \ldots, \delta_{l}, \alpha, \delta_{3}, \ldots, \delta_{k}, \gamma, \delta_{l+2}, \ldots\right),
$$

and let $z$ be equal to $y$ but with the cycle $\sigma$ replaced by $\bar{\sigma}$. We have changed the images of precisely three elements in $\operatorname{supp}(\sigma)$, namely $\delta_{1}, \delta_{k}$ and $\delta_{l}$, and so in the product $x z$ only the images of $\delta_{1}^{x^{-1}}, \delta_{k}^{x^{-1}}$ and $\delta_{l}^{x^{-1}}$ have been changed from those in $x y$. Therefore $x z$ contains the cycle

$$
\left(\delta_{1}^{x^{-1}}, \beta, \ldots, \delta_{k}^{x^{-1}}, \gamma, \ldots, \delta_{l}^{x^{-1}}, \alpha, \ldots\right),
$$

and we have $\left|\mathcal{O}_{x z}(\alpha)\right|=\left|\mathcal{O}_{x y}(\alpha)\right|+\left|\mathcal{O}_{x y}(\beta)\right|+\left|\mathcal{O}_{x y}(\gamma)\right|$. This proves statement (i).

Now suppose that $\gamma \in \mathcal{O}_{x y}(\beta)$, so we may assume $x y$ contains the cycles

$$
\left(\delta_{1}^{x^{-1}}, \alpha, \ldots\right)\left(\delta_{k}^{x^{-1}}, \beta, \ldots, \delta_{l}^{x^{-1}}, \gamma, \ldots\right) .
$$

Once more we set

$$
\bar{\sigma}=\left(\delta_{1}, \beta, \delta_{k+2}, \ldots, \delta_{l}, \alpha, \delta_{3}, \ldots, \delta_{k}, \gamma, \delta_{l+2}, \ldots\right),
$$

and let $z$ be equal to $y$ but with the cycle $\sigma$ replaced by $\bar{\sigma}$. As previously, in the product $x z$ only the images of $\delta_{1}^{x^{-1}}, \delta_{k}^{x^{-1}}$ and $\delta_{l}^{x^{-1}}$ have been changed from those in $x y$. Hence $x z$ contains the cycle

$$
\left(\delta_{1}^{x^{-1}}, \beta, \ldots, \delta_{l}^{x^{-1}}, \alpha, \ldots\right)
$$

and so $\left|\mathcal{O}_{x z}(\alpha)\right|=\left|\mathcal{O}_{x y}(\alpha)\right|+\left|\beta \cdots \delta_{l}^{x^{-1}}\right|_{x y}$, where $\left|\beta \cdots \delta_{l}^{x^{-1}}\right|_{x y}$ denotes the distance between $\beta$ and $\delta_{l}^{x^{-1}}$ in the relevant cycle of $x y$ (reading inclusively from left to right). As a consequence, we have that $\left|\mathcal{O}_{x z}(\gamma)\right|=\left|\mathcal{O}_{x y}(\gamma)\right|-$ $\left|\beta \cdots \delta_{l}^{x^{-1}}\right|_{x y}$. This proves statement $(i i)$.

Now let $\left|\mathcal{O}_{y}(\alpha) \cap\left(\mathcal{O}_{x y}(\alpha) \cup \mathcal{O}_{x y}(\beta)\right)\right|=m$, where $m \geq 4$, and suppose without loss of generality that $\beta$ is the first element of $\mathcal{O}_{x y}(\beta)$ which we encounter when reading from left to right in the cycle $\sigma$, starting at $\alpha$. If there exists $\mu_{1} \in \mathcal{O}_{y}(\beta)$ such that $\mu_{1} \neq \alpha$ but $\mu_{1} \in \mathcal{O}_{x y}(\alpha)$, we may apply (ii) to find an element $y_{1} \in G$ such that $\left|\mathcal{O}_{x y_{1}}(\beta)\right|>\left|\mathcal{O}_{x y}(\beta)\right|$ and $\alpha \notin \mathcal{O}_{x y_{1}}(\beta)$ (here $\beta$ is playing the role of $\alpha$ in the application of $(i i))$. Now, if there exists $\mu_{2} \in \mathcal{O}_{y_{1}}(\beta)$ such that $\mu_{2} \neq \alpha$ but $\mu_{2} \in \mathcal{O}_{x y_{1}}(\alpha)$, we may apply $(i i)$ again to find $y_{2}$ such that $\left|\mathcal{O}_{x y_{2}}(\beta)\right|>\left|\mathcal{O}_{x y_{1}}(\beta)\right|$ and $\alpha \notin \mathcal{O}_{x y_{2}}(\beta)$. Continuing in this way, we eventually find an element $y_{s}$ such that the only element of $\mathcal{O}_{y_{s-1}}(\beta) \cap\left(\mathcal{O}_{x y_{s-1}}(\alpha) \cup \mathcal{O}_{x y_{s-1}}(\beta)\right)$ which lies outside $\mathcal{O}_{x y_{s}}(\beta)$ is $\alpha$.

There are now $m-1$ elements of $\mathcal{O}_{x y_{s}}(\beta)$ which also lie in $\mathcal{O}_{y_{s}}(\alpha)$. We wish to apply (ii) once more, with different choices for $\beta$ and $\gamma$, which we label $\beta^{\prime}$ and $\gamma^{\prime}$. We have $m-2$ choices of element $\beta^{\prime}$ to play the role of $\beta$ in the application of $(i i)$. After choosing a $\beta^{\prime}$, the only requirement for choosing an element $\gamma^{\prime} \in \mathcal{O}_{x y_{s}}\left(\beta^{\prime}\right)$ is that $\gamma^{\prime}$ lies between $\beta^{\prime}$ and $\alpha$ in the relevant cycle of 
$y_{s}$, when we read from left to right starting at $\beta^{\prime}$. So for the first possible $\beta^{\prime}$ (reading from left to right in $y_{s}$ starting at $\alpha$ ), there are $m-2$ choices for $\gamma^{\prime}$. For the second possible $\beta^{\prime}$ there are $m-3$ choices for $\gamma^{\prime}$, and so on. Therefore the total number of choices $M$ we have is

$$
M=(m-2)+(m-3)+\cdots+2+1=(m-1)(m-2) / 2,
$$

which leads to the elements $z_{1}, \ldots, z_{M}$ as in statement (iii). Moreover, after fixing a $\beta^{\prime}$, each subsequent choice of $\gamma^{\prime}$ leads to a different value of $c$ in (ii). As noted, we had $m-2$ choices for $\gamma^{\prime}$ when $\beta^{\prime}$ was our first possible choice. Let $c_{1}, \ldots, c_{m-2}$ be the values of $c$ arising from these choices, labelled so that $c_{j}<c_{i}$ if $j<i$. Suppose $x y_{s}$ contains the cycle

$$
\left(\beta_{1}, \ldots, \delta_{\gamma_{j}}^{x^{-1}}, \gamma_{j}, \ldots, \delta_{\gamma_{i}}^{x^{-1}}, \gamma_{i}, \ldots\right)
$$

and that $c_{i}=\left|\beta_{1} \cdots \delta_{\gamma_{i}}^{x^{-1}}\right|_{x y_{s}}$ and $c_{j}=\left|\beta_{1} \cdots \delta_{\gamma_{j}}^{x^{-1}}\right|_{x y_{s}}$. Then if we choose $\beta^{\prime}=\gamma_{j}$ and $\gamma^{\prime}=\gamma_{i}$ when applying (ii), we get

$$
c=\left|\gamma_{j} \cdots \delta_{\gamma_{i}}^{x^{-1}}\right|_{x y_{s}}=\left|\beta_{1} \cdots \delta_{\gamma_{i}}^{x^{-1}}\right|_{x y_{s}}-\left|\beta_{1} \cdots \delta_{\gamma_{j}}^{x^{-1}}\right|_{x y_{s}}=c_{i}-c_{j} .
$$

As every possible value of $c$ must arise in this way, we see that the penultimate statement in (iii) holds. The final statement follows since $c_{1}, \ldots, c_{m-2}$ are all distinct. This completes the proof.

Let us illustrate Lemma 3.7 with a brief example. Suppose $G=\operatorname{Sym}(16)$,

$$
x=(1,9,8,14,15,4,5)(2,3,6,7,10,11,16)
$$

and

$$
y=\lambda \sigma=(1,2,3,4,5,6,7)(8,9,10,11,12,13,14) .
$$

Then

$$
x y=(1,10,12,13,14,15,5,2,4,6)(3,7,11,16)(8)(9) .
$$

Let $\alpha=8$ and $\beta=10$. Then

$$
\mathcal{O}_{y}(8) \cap\left(\mathcal{O}_{x y}(8) \cup \mathcal{O}_{x y}(10)\right)=\{8,10,12,13,14\},
$$

and (iii) tells us there exist $4 \cdot 3 / 2=6$ distinct elements $z_{1}, \ldots, z_{6}$, where for each $i$ the orbits of $\left\langle z_{i}\right\rangle$ are the same as those of $\langle y\rangle$, and that $\left\{\left|\mathcal{O}_{x z_{i}}(8)\right|: 1 \leq i \leq 6\right\}$ has cardinality at least $5-2=3$. Explicitly, we apply (ii) by adjusting the cycle $\sigma$ of $y$ to the following:

$$
\begin{aligned}
& \sigma_{1}=(10,11,8,9,12,13,14) \\
& \sigma_{2}=(10,11,12,8,9,13,14) \\
& \sigma_{3}=(10,11,12,13,8,9,14) \\
& \sigma_{4}=(12,8,9,10,11,13,14) \\
& \sigma_{5}=(12,13,8,9,10,11,14) \\
& \sigma_{6}=(13,8,9,10,11,12,14) .
\end{aligned}
$$


Setting $z_{i}=\lambda \sigma_{i}$, for $1 \leq i \leq 6$, we have

$$
\begin{aligned}
& x z_{1}=(1,12,13,14,15,5,2,4,6)(3,7,11,16)(8,10)(9) \\
& x z_{2}=(1,13,14,15,5,2,4,6)(3,7,11,16)(8,10,12)(9) \\
& x z_{3}=(1,14,15,5,2,4,6)(3,7,11,16)(8,10,12,13)(9) \\
& x z_{4}=(1,10,13,14,15,5,2,4,6)(3,7,11,16)(8,12)(9) \\
& x z_{5}=(1,10,14,15,5,2,4,6)(3,7,11,16)(8,12,13)(9) \\
& x z_{6}=(1,10,12,14,15,5,2,4,6)(3,7,11,16)(8,13)(9),
\end{aligned}
$$

and we see that $\left\{\left|\mathcal{O}_{x z_{i}}(8)\right| \mid 1 \leq i \leq 6\right\}=\{2,3,4\}$.

Notice that in Lemma 3.7, the elements $x$ and $y$ need not be $G$-conjugate. Also, the proof can be easily modified to give a corresponding result regarding the adjustment of the orbits of $\langle x\rangle$. We are now in a position to deal with the case of single $p$-cycles.

Proof of Theorem 1.2. When $p=3$ the result is clear, so assume $p \geq 5$. Let $t=(1,2, \ldots, p)$ be our base point, and let $x \in X$. Suppose that $t$ and $x$ are disjoint cycles. Then by Proposition $3.6, d(t, x) \leq 2$. So we may assume $|\operatorname{supp}(t) \cup \operatorname{supp}(x)|<2 p$.

Write $y=x^{-1}$. Clearly $y$ is adjacent to $x$ in $\mathcal{C}_{p^{\prime}}(G, X)$, but suppose $t$ and $y$ are not adjacent. Then we must have $t y=\sigma \mu$, where $\sigma$ is a $p$-cycle disjoint from $\mu$, a product of cycles of length less than $p$.

Suppose we have $\operatorname{supp}(t)=\operatorname{supp}(\sigma)$. Then we claim that $\operatorname{supp}(t)=\operatorname{supp}(y)$. Indeed, suppose not. Then $\operatorname{since} \operatorname{supp}(y)=\operatorname{supp}\left(x^{-1}\right)=\operatorname{supp}(x)$, and $\operatorname{supp}(x) \cap$ $\operatorname{supp}(t) \neq \emptyset$, there exists $\alpha \in \operatorname{supp}(y)$ such that $\alpha \notin \operatorname{supp}(t)$ but $\alpha^{y} \in \operatorname{supp}(t)$. Then $\alpha^{t y}=\alpha^{y} \in \operatorname{supp}(t)=\operatorname{supp}(\sigma)$, and since $\sigma$ and $\mu$ are disjoint, this implies that $\alpha^{t y}$ is fixed by $\mu$. We therefore have

$$
\alpha=\left(\alpha^{t y}\right)^{y^{-1} t^{-1}}=\left(\alpha^{t y}\right)^{\mu^{-1} \sigma^{-1}}=\left(\alpha^{t y}\right)^{\sigma^{-1}},
$$

and hence $\alpha \in \operatorname{supp}(\sigma)=\operatorname{supp}(t)$, a contradiction, and the claim holds. Now Theorem 3.4 tells us that $d(t, x) \leq 2$.

So let $\beta \in \operatorname{supp}(y) \cap \operatorname{supp}(\sigma)$. Suppose there exist distinct $\gamma, \delta \in \operatorname{supp}(y)$ such that $\gamma, \delta \notin \operatorname{supp}(\sigma)$. Then we may apply Lemma 3.7(i) or (ii) to obtain $z \in X$ such that $t z$ contains a cycle of length greater than $p$. Hence $t$ and $z$ are adjacent in $\mathcal{C}_{p^{\prime}}(G, X)$. But $\langle z\rangle$ and $\langle x\rangle$ have the same orbits, and at least one element of the $p$-cycle of $x$ is fixed by $z x$ (since $x=y^{-1}$, and $z$ was obtained from $y$ by changing the images of at most three points). Hence $z$ is also adjacent to $x$. Thus $d(t, x) \leq 2$ in this case.

Now suppose $\gamma \in \operatorname{supp}(y)$ is the only such point for which $\gamma \notin \operatorname{supp}(\sigma)$. Then we may apply Lemma 3.7 to obtain $z$ such that $\left|\mathcal{O}_{t z}(\gamma)\right|>\left|\mathcal{O}_{t y}(\gamma)\right|$. Furthermore, by Lemma 3.7(iii) we have enough freedom of choice in choosing $z$ to ensure that $\left|\mathcal{O}_{t z}(\gamma)\right| \neq p$. Hence $t$ and $z$ are adjacent, as are $z$ and $x$. Therefore $d(t, x) \leq 2$ in this final case, and the proof is complete. 
Corollary 3.8. Let $G=\operatorname{Sym}(n)$, where $n \geq 4$, with $X$ the conjugacy class of a p-cycle, where $p \geq 3$ is prime. Suppose $t, x \in X$ are adjacent in $\mathcal{C}_{p^{\prime}}(G, X)$. Then there exists $z \in X$ such that $d(t, z)=1$ and $d(z, x)=1$.

Proof. Set $y=x^{-1}$. If $y$ is adjacent to $t$ then clearly we may let $z=y$. Otherwise we may argue as in the proof of Theorem 1.2 to find a suitable $z$, by adjusting $y$ using Lemma 3.7 .

When addressing the case of products of pairwise disjoint $p$-cycles, we wish to decompose elements into pieces which are in some sense minimal, and thus easier to work with. This motivates what follows.

Definition 3.9. Let $G=\operatorname{Sym}(n)$, with $x, y \in G$ elements of order of prime order $p$, not necessarily $G$-conjugate. Write $x=x_{1} x_{2} \cdots x_{r}$ and $y=y_{1} y_{2} \cdots y_{s}$ as products of pairwise disjoint $p$-cycles, and denote by $A$ the set of non-trivial orbits of $\langle x\rangle$ and $\langle y\rangle$. We say the pair $(x, y)$ is disentangled if we can write $A=B \cup C$, where $B$ and $C$ are nonempty subsets of $A$ such that

$$
\left(\bigcup_{b \in B} b\right) \cap\left(\bigcup_{c \in C} c\right)=\emptyset
$$

If this is not possible we say $(x, y)$ is tangled.

If we allow the 'empty permutation', which we denote by $(\emptyset)$, then for every pair $(x, y)$ there exists a decomposition $x=x^{(1)} \cdots x^{(k)}, y=y^{(1)} \cdots y^{(k)}$ such that each pair $\left(x^{(i)}, y^{(i)}\right)$ is tangled.

To illustrate the above we give some examples. Suppose that $G=\operatorname{Sym}(30)$, and let

$$
x=(1,2,3,4,5)(6,7,8,9,10)(11,12,13,14,15)(16,17,18,19,20)
$$

and

$$
y=(1,3,6,8,21)(2,9,10,23,28)(11,22,12,14,16)(18,29,19,26,27) .
$$

Then $(x, y)$ is disentangled, with decomposition

\begin{tabular}{|c|c|c|}
\hline$i$ & $x^{(i)}$ & $y^{(i)}$ \\
\hline 1 & $(1,2,3,4,5)(6,7,8,9,10)$ & $(1,3,6,8,21)(2,9,10,23,28)$ \\
2 & $(11,12,13,14,15)(16,17,18,19,20)$ & $(11,22,13,14,16)(18,29,19,26,27)$ \\
\hline
\end{tabular}

Now let

$$
y=(1,7,4,12,15)(26,28,22,30,29) .
$$

Then $(x, y)$ is again disentangled, with decomposition

\begin{tabular}{|c|c|c|}
\hline$i$ & $x^{(i)}$ & $y^{(i)}$ \\
\hline 1 & $(1,2,3,4,5)(6,7,8,9,10)(11,12,13,14,15)$ & $(1,7,4,12,15)$ \\
2 & $(16,17,18,19,20)$ & $(\emptyset)$ \\
3 & $(\emptyset)$ & $(26,28,22,30,29)$ \\
\hline
\end{tabular}


Lemma 3.10. Let $G=\operatorname{Sym}(\Omega)$, and let $x, y \in G$ be elements of order $p \geq 3$ such that $(x, y)$ is tangled. Let $|\operatorname{supp}(x) \cup \operatorname{supp}(y)|=m$, and suppose that $x y$ is not an $m$-cycle. Then for any cycle $\sigma$ in the product $x y$, we may find a cycle $\rho$ of either $x$ or $y$ with $\alpha, \beta \in \operatorname{supp}(\rho)$ such that $\alpha \in \operatorname{supp}(\sigma)$ but $\beta \notin \operatorname{supp}(\sigma)$.

Proof. First we write $x$ and $y$ as products of pairwise disjoint cycles, thus $x=$ $x_{1} x_{2} \ldots x_{s}$ and $y=y_{1} y_{2} \ldots y_{r}$. For a contradiction suppose the result does not hold for some cycle $\sigma$ of $x y$. Then if $\mathcal{O}$ is any orbit of $\langle x\rangle$ or $\langle y\rangle$, then either $\mathcal{O}$ is disjoint from $\operatorname{supp}(\sigma)$, or $\mathcal{O} \subseteq \operatorname{supp}(\sigma)$. Thus if $A$ is the set of orbits of $\langle x\rangle$ and $\langle y\rangle$, then we may write $A=B \cup C$, where $B$ is the set of orbits which lie in $\operatorname{supp}(\sigma)$ and $C=A \backslash B$. Clearly $B$ is nonempty and, since $x y$ is not an $m$-cycle, $C$ must also be nonempty. Since by the above observation we have

$$
\left(\bigcup_{b \in B} b\right) \cap\left(\bigcup_{c \in C} c\right)=\emptyset
$$

this implies the pair $(x, y)$ is disentangled, which is the desired contradiction.

We now begin our attack on Theorem 1.3 and Theorem 1.4, and work under the following hypothesis:

Hyposthesis 3.11. Let $G=\operatorname{Sym}(n)$, with $x, y \in G$ such that $x=x_{1} x_{2} \ldots x_{r}$ and $y=y_{1} y_{2} \ldots y_{s}$ are products of pairwise disjoint $p$-cycles, where $p \geq 7$ is an odd prime. Furthermore, suppose that $(x, y)$ is tangled, and that $r, s<\sqrt{p}$.

Lemma 3.12. Suppose Hypothesis 3.11 holds, and additionally that $\mid \operatorname{supp}(x) \cup$ $\operatorname{supp}(y) \mid=k p$ for some $k \in \mathbb{N}$, and that $x y$ is a $k p$-cycle. Then there exist elements $x^{\prime} \in x^{G}, y^{\prime} \in y^{G}$ such that $\langle x\rangle$ and $\left\langle x^{\prime}\right\rangle$ have the same orbits on $\Omega,\langle y\rangle$ and $\left\langle y^{\prime}\right\rangle$ have the same orbits on $\Omega$, and the order of the product $x^{\prime} y^{\prime}$ is coprime to $p$.

Proof. We first show that since $r, s<\sqrt{p},(x, y)$ is tangled and $\mid \operatorname{supp}(x) \cup$ $\operatorname{supp}(y) \mid=k p$, there must exist cycles $\lambda_{x}, \lambda_{y}$, of $x$ and $y$ respectively, with $\left|\operatorname{supp}\left(\lambda_{x}\right) \cap \operatorname{supp}\left(\lambda_{y}\right)\right| \geq 2$. For suppose this is not the case. Then

$$
|\operatorname{supp}(x) \cup \operatorname{supp}(y)| \geq r p+s p-s r>(r+s) p-\sqrt{p} \cdot \sqrt{p}=(r+s-1) p .
$$

On the other hand, again since $(x, y)$ is tangled, we have that

$$
|\operatorname{supp}(x) \cup \operatorname{supp}(y)|<(r+s) p .
$$

However, by assumption, $|\operatorname{supp}(x) \cup \operatorname{supp}(y)|$ is a multiple of $p$, so this is a contradiction. Therefore we may choose $\alpha, \beta \in \operatorname{supp}\left(\lambda_{x}\right) \cap \operatorname{supp}\left(\lambda_{y}\right)$ with $\alpha \neq \beta$. We may write

$$
\lambda_{x}=\left(\delta_{1}, \alpha, \delta_{3}, \ldots, \delta, \beta, \delta_{k+2}, \ldots\right) .
$$

Then we construct an element $x^{\prime} \in x^{G}$, containing a cycle $\lambda_{x^{\prime}}$, by adjusting the position of $\beta$ in the cycle $\lambda_{x}$ so that $\beta=\alpha^{\lambda_{x^{\prime}}}$ (if this is already the case, we set $\left.x^{\prime}=x\right)$. So

$$
\lambda_{x^{\prime}}=\left(\delta_{1}, \alpha, \beta, \delta_{3}, \ldots, \delta, \delta_{k+2}, \ldots\right) .
$$


We now show that

(3.1) when considered as an element of $\operatorname{Sym}(\operatorname{supp}(x) \cup \operatorname{supp}(y)), x^{\prime} y$ is either a single cycle or a product of exactly three cycles.

Firstly, note that if $x=x^{\prime}$, then $x^{\prime} y=x y$ is already a single $k p$-cycle, so assume that this is not the case. Then we have changed the image under $\lambda_{x}$ of exactly three elements, namely $\alpha, \beta$ and $\delta$. So all but these three elements in $\operatorname{supp}\left(x^{\prime} y\right)$ will have the same image under $x^{\prime} y$ as under $x y$. In view of this, $x^{\prime} y$ cannot be a product of more than three cycles. Suppose $\alpha^{x y}=\gamma_{1}, \beta^{x y}=\gamma_{2}$ and $\delta^{x y}=\gamma_{3}$. We can therefore write either

(3.1.1) $x y=\left(\alpha, \gamma_{1}, \ldots, \beta, \gamma_{2}, \ldots, \delta, \gamma_{3}, \ldots\right)$, or

$(3.1 .2) x y=\left(\alpha, \gamma_{1}, \ldots, \delta, \gamma_{3}, \ldots, \beta, \gamma_{2}, \ldots\right)$.

(Note that it might be the case that $\{\alpha, \beta, \delta\} \cap\left\{\gamma_{1}, \gamma_{2}, \gamma_{3}\right\} \neq \emptyset$ ).

Since in both (3.1.1) and (3.1.2) we have $\delta^{x}=\beta$ and $\delta^{x y}=\gamma_{3}$, it must be that $\beta^{y}=\gamma_{3}$. Now, as $\alpha^{x^{\prime}}=\beta$, we deduce that $\alpha^{x^{\prime} y}=\gamma_{3}$. Consequently, we must have $\beta^{x^{\prime} y}=\gamma_{1}$ and $\delta^{x^{\prime} y}=\gamma_{2}$. Hence if (3.1.1) holds we have

$$
x^{\prime} y=\left(\alpha, \gamma_{3}, \ldots\right)\left(\delta, \gamma_{2}, \ldots\right)\left(\beta, \gamma_{1}, \ldots\right) .
$$

On the other hand, if $x y$ is as in (3.1.2), we see that

$$
x^{\prime} y=\left(\alpha, \gamma_{2}, \ldots, \beta, \gamma_{1}, \ldots, \delta, \gamma_{3}, \ldots\right) \text {. }
$$

Thus (3.1) holds.

Now we construct an element $y^{\prime} \in y^{G}$ by adjusting the position of $\beta$ in the cycle $\lambda_{y}$ so that $\alpha=\beta^{\lambda_{y^{\prime}}}$. If this is already the case, we set $y^{\prime}=y$ (note that if we set $x=x^{\prime}$ above, then it cannot be the case that $y=y^{\prime}$, since this would imply that $\alpha$ and $\beta$ are fixed points of $x y$, which contradicts their lying in the $k p$-cycle of $x y$ ). We may write

$$
\lambda_{y}=\left(\epsilon_{1}, \alpha, \epsilon_{3}, \ldots, \epsilon_{l}, \beta, \epsilon, \ldots\right)
$$

and

$$
\lambda_{y^{\prime}}=\left(\epsilon_{1}, \beta, \alpha, \epsilon_{3}, \ldots, \epsilon_{l}, \epsilon, \ldots\right) .
$$

Next, we show that

(3.2) when considered as an element of $\operatorname{Sym}(\operatorname{supp}(x) \cup \operatorname{supp}(y)), x^{\prime} y^{\prime}$ is a product of exactly three cycles.

If $x^{\prime} y$ is a single cycle, then a similar argument to that above shows that $x^{\prime} y^{\prime}$ is either a single cycle, or a product of exactly three cycles. But $\alpha$ is fixed by $x^{\prime} y^{\prime}$, so $x^{\prime} y^{\prime}$ cannot be a single cycle, and hence the result holds in this case. We may therefore assume that $x^{\prime} y$ is a product of exactly three cycles. 
In our rearrangement of $\lambda_{y}$ we have changed the preimage under $\lambda_{y}$ of exactly three elements, which are $\alpha, \beta$ and $\epsilon$. Suppose that $\zeta_{1}^{x^{\prime} y}=\alpha, \zeta_{2}^{x^{\prime} y}=\beta$ and $\zeta_{3}^{x^{\prime} y}=\epsilon$. We may write

$$
x^{\prime} y=\left(\zeta_{1}, \alpha, \gamma_{3}, \ldots\right)\left(\delta, \gamma_{2}, \ldots\right)\left(\zeta_{2}, \beta, \gamma_{1}, \ldots\right) .
$$

As a consequence of our rearrangement, the images of $\zeta_{1}, \zeta_{2}$, and $\zeta_{3}$ are also changed under $x^{\prime} y^{\prime}$. As $\zeta_{3}^{x^{\prime} y}=\epsilon$, and $\beta^{y}=\epsilon$, we must have that $\zeta_{3}^{x^{\prime}}=\beta$. Since $\beta^{y^{\prime}}=\alpha$ we have that $\zeta_{3}^{x^{\prime} y^{\prime}}=\alpha$, but $\alpha$ is fixed by $x^{\prime} y^{\prime}$, so it must be that $\zeta_{3}=\alpha$. Consequently, $\epsilon=\gamma_{3}, \zeta_{1}^{x^{\prime} y^{\prime}}=\beta$ and $\zeta_{2}^{x^{\prime} y^{\prime}}=\epsilon$. Since only three elements of $\operatorname{supp}\left(x^{\prime} y\right)$ have different images under $x^{\prime} y^{\prime}$ than under $x^{\prime} y$, we deduce that

$$
x^{\prime} y^{\prime}=(\alpha)\left(\zeta_{1}, \beta, \gamma_{1}, \ldots, \zeta_{2}, \epsilon, \ldots\right)\left(\delta, \gamma_{2}, \ldots\right) .
$$

This proves (3.2).

Thus, when considered as an element of $\operatorname{Sym}(\operatorname{supp}(x) \cup \operatorname{supp}(y)), x^{\prime} y^{\prime}$ is a product of exactly three cycles, one of which is a 1-cycle. If $p$ does not divide the length of either of the other cycles, then $x^{\prime}$ and $y^{\prime}$ satisfy the conclusions of the lemma. So suppose $\sigma_{w p}$ is a cycle of $x^{\prime} y^{\prime}$ of length $w p$ where $1 \leq w<k$, and let $\rho$ be the remaining non-trivial cycle of $x^{\prime} y^{\prime}$. Note that since $|\operatorname{supp}(x) \cup \operatorname{supp}(y)|=$ $k p$, this means that $p$ cannot divide the length of the cycle $\rho$. Since $(x, y)$, and hence $\left(x^{\prime}, y^{\prime}\right)$, is tangled, and $x^{\prime} y^{\prime}$ is not a $k p$-cycle, we may apply Lemma 3.10 to see that there exists some cycle $\lambda$ of either $x^{\prime}$ or $y^{\prime}$ with $\mu, \nu \in \operatorname{supp}(\lambda)$ such that $\mu \in \operatorname{supp}\left(\sigma_{w p}\right)$ but $\nu \notin \operatorname{supp}\left(\sigma_{w p}\right)$.

Without loss of generality suppose $\lambda$ is a cycle of $y^{\prime}$, and suppose we may choose $\nu$ so that $\nu \neq \alpha$. Then since $\mathcal{O}_{x^{\prime} y^{\prime}}(\mu) \cup \mathcal{O}_{x^{\prime} y^{\prime}}(\nu)$ covers all of $\operatorname{supp}(x) \cup$ $\operatorname{supp}(y) \operatorname{except} \alpha$, and $\operatorname{supp}\left(y^{\prime}\right)=\operatorname{supp}(y)$, we have

$$
\left|\mathcal{O}_{y^{\prime}}(\mu) \cap\left(\mathcal{O}_{x^{\prime} y^{\prime}}(\mu) \cup \mathcal{O}_{x^{\prime} y^{\prime}}(\nu)\right)\right| \geq p-1 .
$$

Now apply Lemma 3.7(ii) to construct an element $y^{\prime \prime}$ such that $\left|\mathcal{O}_{x^{\prime} y^{\prime \prime}}(\mu)\right|>$ $\left|\mathcal{O}_{x^{\prime} y^{\prime}}(\mu)\right|$. This will ensure coprimality, unless the element $y^{\prime \prime}$ which we construct yields $x^{\prime} y^{\prime \prime}$ with $\left|\mathcal{O}_{x^{\prime} y^{\prime \prime}}(\mu)\right|=u p$ or $\left|\mathcal{O}_{x^{\prime} y^{\prime \prime}}\left(\nu^{\prime}\right)\right|=v p$, where $1 \leq u, v \leq$ $k-1$, and $\nu^{\prime}$ lies in the other cycle of $x^{\prime} y^{\prime}$ whose length has been adjusted by applying Lemma 3.7(ii). Since, by assumption, we are already in the situation where $\left|\mathcal{O}_{x^{\prime} y^{\prime}}(\mu)\right|=w p$, and applying Lemma 3.7 adjusts the length of this orbit, we deduce that there are $2 k-3$ possible problem cases.

By Lemma 3.7(iii) we have at least $p-3$ choices of $y^{\prime \prime}$ which yield distinct values of $c$ such that $\left|\mathcal{O}_{x^{\prime} y^{\prime \prime}}(\mu)\right|=\left|\mathcal{O}_{x^{\prime} y^{\prime}}(\mu)\right|+c$. Since $(x, y)$ is tangled (so $x$ and $y$ are not disjoint), $2 k-3 \leq 2(r+s-1)-3$. When $p \geq 17$ then $4<\sqrt{p}$, and since $r, s<\sqrt{p}$ we have

$$
2(r+s-1)-3<4 \sqrt{p}-5<p-3 .
$$

The number of problem cases is therefore fewer than the number of possibilities for $c$, so we may choose $y^{\prime \prime}$ to ensure coprimality. When $p=7,11$ or 13 , we may explicitly count the number of problem cases as at most 3,7 and 7 respectively, 
which are less than $p-3$ in each case. So again we may choose $y^{\prime \prime}$ to ensure coprimality.

On the other hand, it may be the case that we are forced to take $\nu=\alpha$. However, we then apply Lemma 3.7(ii) to adjust the lengths of $\mathcal{O}_{x^{\prime} y^{\prime}}(\mu)$ and $\mathcal{O}_{x^{\prime} y^{\prime}}(\alpha)$, and again use Lemma 3.7(iii) in a similar way to that above to show we can ensure coprimality.

We now drop our assumptions on the size of $\operatorname{supp}(x) \cup \operatorname{supp}(y)$ and cycle type of $x y$.

Lemma 3.13. Suppose Hyposthesis 3.11 holds. Then there exists elements $x^{\prime} \in x^{G}, y^{\prime} \in y^{G}$ such that $\left\langle x^{\prime}\right\rangle$, respectively $\left\langle y^{\prime}\right\rangle$, has the same orbits on $\Omega$ as $\langle x\rangle$, respectively $\langle y\rangle$, and the product $x^{\prime} y^{\prime}$ has order coprime to $p$.

Proof. If $x y$ has order coprime to $p$, then clearly setting $x^{\prime}=x, y^{\prime}=y$ satisfies the lemma, so assume this is not the case. As in the proof of Lemma 3.12 we consider $x y$ as an element of $\operatorname{Sym}(\operatorname{supp}(x) \cup \operatorname{supp}(y))$. Firstly, suppose that $x y=\sigma_{k p} \prod_{i=1}^{l} \rho_{i}$, where $\sigma_{k p}$ is a cycle of length $k p$, and $\rho_{1}, \ldots, \rho_{l}$ are cycles of length coprime to $p$ (possibly 1-cycles). If no such $\rho_{i}$ exist, then $x y$ is a $k p$-cycle and $|\operatorname{supp}(x) \cup \operatorname{supp}(y)|=k p$, so we may apply Lemma 3.12 to obtain suitable elements $x^{\prime}$ and $y^{\prime}$. Thus we may assume there is at least one $\rho_{i}$. By Lemma 3.10 there exists a cycle $\lambda$ of either $x$ or $y$, with $\alpha, \beta \in \operatorname{supp}(\lambda)$ such that $\alpha \in \operatorname{supp}\left(\sigma_{k p}\right)$ and $\beta \notin \operatorname{supp}\left(\sigma_{k p}\right)$. Without loss of generality we suppose that $\lambda$ is a cycle of $y$, and that $\beta \in \operatorname{supp}\left(\rho_{1}\right)$.

We now apply Lemma 3.7(i) (if possible) to increase the length of $\sigma_{k p}$ by 'merging' it with some of the $\rho_{i}$, and we do this as many times as we can until it becomes impossible to apply (i). We therefore get an element $y^{\prime}$ such that either $x y^{\prime}$ is a single cycle, or all elements of $\lambda$ which do not lie in $\mathcal{O}_{x y^{\prime}}(\alpha)$ lie in only one other orbit of $\left\langle x y^{\prime}\right\rangle$, which without loss we assume to be $\mathcal{O}_{x y^{\prime}}(\beta)$. In the case where $x y^{\prime}$ is a single cycle, we either have coprimality, or if $p$ divides this cycle length we may apply Lemma 3.12 to establish the result. In the latter case we either have coprimality, or at least one of $\left|\mathcal{O}_{x y^{\prime}}(\alpha)\right|$ and $\left|\mathcal{O}_{x y^{\prime}}(\beta)\right|$ is divisible by $p$. Notice that $\left|\mathcal{O}_{y^{\prime}}(\alpha) \cap\left(\mathcal{O}_{x y^{\prime}}(\alpha) \cup \mathcal{O}_{x y^{\prime}}(\beta)\right)\right|=p$. We now apply Lemma 3.7(ii) to adjust the lengths of these two cycles of $x y^{\prime}$. Note that no other cycles of $x y^{\prime}$ are affected by this. This will ensure coprimality unless the element $y^{\prime \prime}$ which we construct yields product $x y^{\prime \prime}$ with $\left|\mathcal{O}_{x y^{\prime \prime}}(\alpha)\right|=u p$ or $\left|\mathcal{O}_{x y^{\prime \prime}}(\gamma)\right|=v p$, where $1 \leq u, v<(r+s)$, and $\mathcal{O}_{x y^{\prime \prime}}(\gamma)$ is the other orbit whose length we affect. By Lemma 3.7(iii) we have at least $(p-1)(p-2) / 2$ choices of element $y^{\prime \prime}$ for which $\left|\mathcal{O}_{x y^{\prime \prime}}(\alpha)\right|=\left|\mathcal{O}_{x y^{\prime}}(\alpha)\right|+c_{y^{\prime \prime}}$ (where $c_{y^{\prime \prime}}$ depends on our choice of $y^{\prime \prime}$, with at least $p-2$ distinct possibilities).

Suppose that both $\left|\mathcal{O}_{x y^{\prime}}(\alpha)\right|$ and $\left|\mathcal{O}_{x y^{\prime}}(\beta)\right|$ are divisible by $p$. Then if we construct $y^{\prime \prime}$ so that $\left|\mathcal{O}_{x y^{\prime \prime}}(\alpha)\right|$ is a mutiple of $p$, then $\left|\mathcal{O}_{x y^{\prime \prime}}(\gamma)\right|$ must also be a multiple of $p$. Since we have assumed that we start with $\left|\mathcal{O}_{x y^{\prime}}(\alpha)\right|$ a multiple of $p$, there are $(r+s-1)-1=r+s-2$ problem cases in this situation. But for $p \geq 7, r+s-2<p-2$, so we can choose $y^{\prime \prime}$ so that neither $\left|\mathcal{O}_{x y^{\prime \prime}}(\alpha)\right|$ nor $\left|\mathcal{O}_{x y^{\prime \prime}}(\gamma)\right|$ is divisible by $p$, thus ensuring coprimality. 
Now suppose that only one of $\left|\mathcal{O}_{x y^{\prime}}(\alpha)\right|$ and $\left|\mathcal{O}_{x y^{\prime}}(\beta)\right|$ is divisible by $p$. Without loss we assume that $\left|\mathcal{O}_{x y^{\prime}}(\alpha)\right|=w p$ for some $w \in \mathbb{N}$, and that $\left|\mathcal{O}_{x y^{\prime}}(\beta)\right|=l$ where $l \in \mathbb{N}$ is coprime to $p$. When applying Lemma 3.7(ii), we will ensure coprimality unless $c=a p$ or $c=b(p-l)$, where there are at most $r+s-1$ possibilities each for $a, b \in \mathbb{N}$. There are a possible $2(r+s-1)-1=2(r+s)-3$ problem cases here. Let $\left\{c_{1}, c_{2}, \ldots, c_{p-2}\right\}$ be a set of $p-2$ distinct values of $c$ we can guarantee by Lemma 3.7(iii), ordered so that $c_{i}>c_{j}$ when $i>j$, and suppose that for each $i$ either $c_{i}=a p$ for some $a$ or $c_{i}=b(p-l)$ for some $b$. Since $r+s-1<p-2$, it must be the case that $\left\{c_{1}, c_{2}, \ldots, c_{p-2}\right\}$ includes both a multiple of $p$ and a multiple of $p-l$. But since $p$ and $p-l$ are coprime, and by Lemma 3.7(iii) the set of possible values for $c$ is

$$
\left\{c_{i}-c_{j} \mid 0 \leq j<i \leq p-2\right\},
$$

we see there must in fact be at least $2 p-5$ distinct choices for $c$, which ensures coprimality.

Now suppose that $x y=\sigma_{1} \ldots \sigma_{m} \prod_{i=1}^{l} \rho_{i}$, where $\sigma_{1}, \ldots, \sigma_{m}$ are cycles with lengths divisible by $p$, and $m \geq 2$. By Lemma 3.10 there exists a cycle $\lambda$ of either $x$ or $y$, with $\alpha, \beta \in \operatorname{supp}(\lambda)$ such that $\alpha \in \operatorname{supp}\left(\sigma_{1}\right)$ and $\beta \notin \operatorname{supp}\left(\sigma_{1}\right)$. Without loss of generality we suppose that $\lambda$ is a cycle of $y$. As in the previous case we apply Lemma 3.7(i) (if possible) to increase the length of $\sigma_{1}$. Again, we do this multiple times until it becomes impossible to apply Lemma 3.7(i). Then we get an element $y^{\prime}$ such that either the number of cycles with length divisible by $p$ in $x y^{\prime}$ is less than the number in $x y$, or all elements of $\lambda$ which do not lie in $\mathcal{O}_{x y^{\prime}}(\alpha)$ lie in only one other orbit of $x y^{\prime}$, which without loss we assume to be $\mathcal{O}_{x y^{\prime}}(\beta)$. In the former case, by induction the lemma holds for the pair $\left(x, y^{\prime}\right)$. But since $\langle y\rangle$ and $\left\langle y^{\prime}\right\rangle$ have the same orbits on $\Omega$, this implies that the lemma also holds for $(x, y)$. In the latter case, then as previously we may apply Lemma 3.7(ii) to adjust the lengths of $\mathcal{O}_{x y^{\prime}}(\alpha)$ and a subsequent orbit $\mathcal{O}_{x y^{\prime}}(\gamma)$. Lemma 3.7(iii) tells us that we can construct an element $y^{\prime \prime}$ such that for $x y^{\prime \prime}$ neither $\left|\mathcal{O}_{x y^{\prime \prime}}(\alpha)\right|$ nor $\left|\mathcal{O}_{x y^{\prime \prime}}(\gamma)\right|$ is divisible by $p$. Thus the number of cycles of $x y^{\prime \prime}$ with length divisible by $p$ is less than that of $x y$. By induction the lemma holds for $\left(x, y^{\prime \prime}\right)$, whence it also holds for $(x, y)$.

Lemma 3.14. Let $(x, y)$ be a tangled pair, with $x, y \neq(\emptyset)$, and suppose that $x$ contains more $p$-cycles than $y$. Then there exists a cycle $\lambda$ of $x$ such that $\left(x \lambda^{-1}, y\right)$ is still a tangled pair.

Proof. Since $(x, y)$ is tangled, and $x$ contains more cycles than $y$, there must exist cycles $\lambda$ and $\rho$ of $x$ such that for every cycle of $y$ with which $\lambda$ has a nonempty intersection, $\rho$ also has a non-empty intersection. But now if $\left(x \lambda^{-1}, y\right)$ were disentangled, then $(x, y)$ would also be disentangled, a contradiction. Thus $\left(x \lambda^{-1}, y\right)$ is tangled.

We have reached the point where we can prove Theorem 1.3, which we now restate. 
Theorem 3.15. Suppose that $G=\operatorname{Sym}(n)$ and $X$ is the $G$-conjugacy class of elements of cycle type $p^{r}$, where $p$ is an odd prime. If $r<\sqrt{p}$, then $\operatorname{Diam}\left(\mathcal{C}_{p^{\prime}}(G, X)\right) \leq 5$.

Proof. When $p=3$ we must have $r=1$, so we may apply Theorem 1.2 to see that $\operatorname{Diam}\left(\mathcal{C}_{3^{\prime}}(G, X)\right) \leq 2$, where $X$ is the unique $G$-conjugacy class of 3cycles. Now suppose that $p=5$, so $r=1$ or 2 . When $r=1$ we can again apply Theorem 1.2 to show the result holds in this case. Assume then that $r=2$. Let $t \in X$ be our base point, and let $x \in X$, where $X=t^{G}$. Clearly we have $10 \leq|\operatorname{supp}(t) \cup \operatorname{supp}(x)| \leq 20$. Using MAGMA [8] and the class structure constants described in Section 2, it is straightforward to verify that for $10 \leq$ $m \leq 20, \operatorname{Diam}\left(\mathcal{C}_{5^{\prime}}\left(\operatorname{Sym}(m), X^{\prime}\right)=2\right.$, where $X^{\prime}$ is the $\operatorname{Sym}(m)$-conjugacy class of elements with cycle type $5^{2}$. (This is done by calculating that $\left|\Delta_{1}(t)\right|>\left|X^{\prime}\right| / 2$ in each case, and applying Lemma 2.1). Consequently there exists a path of length 2 between $t$ and $x$ in $\mathcal{C}_{5^{\prime}}(G, X)$.

We may therefore proceed on the assumption that $p \geq 7$. Assume $t=$ $t_{1} \ldots t_{k}, x=x_{1} \ldots x_{k}$ is a decomposition of $(t, x)$ into tangled pairs. Note that some of these pairs may be of the form $\left(t_{i},(\emptyset)\right)$ or $\left((\emptyset), x_{i}\right)$. Suppose there are $m_{1}$ such pairs $\left(t_{i},(\emptyset)\right)$ and $m_{2}$ such pairs $\left((\emptyset), x_{i}\right)$, and without loss of generality assume that $m_{2} \geq m_{1}$. By pairing up such cycles, we can get $m_{1}$ pairs $\left(t_{i}, x_{j}\right)$ of disjoint $p$-cycles, which leaves us with $m_{2}-m_{1}$ cycles $x_{j}$ which have not yet been paired up. Note that the support of any one of these cycles intersects in the empty set with the remainder of the support of $t$ and $x$. For each such $x_{j}$, choose a tangled pair $\left(t_{i}, x_{i}\right)$ for which $t_{i}$ has more cycles than $x_{i}$ (such a pair must exist since $t$ and $x$ have the same cycle type), and remove a cycle $\sigma$ from $t_{i}$ in such a way that $\left(t_{i} \sigma^{-1}, x_{i}\right)$ remains tangled (this is possible by Lemma 3.14). Thus $\left(\sigma, x_{j}\right)$ is a pair of disjoint $p$-cycles. In this way we get a new decomposition $t=t_{1} \ldots t_{l} t_{l+1} \ldots t_{v}, x=x_{1} \ldots x_{l} x_{l+1} \ldots x_{v}$ of $(t, x)$, where $\left(t_{i}, x_{i}\right)$ is tangled for $1 \leq i \leq l$ and consists of two disjoint $p$-cycles for $l+1 \leq i \leq v$.

By Lemma 3.13, for each tangled pair $\left(t_{i}, x_{i}\right)$ there exist elements $t_{i}^{\prime}, x_{i}^{\prime}$ such that $\left\langle t_{i}^{\prime}\right\rangle$, respectively $\left\langle x_{i}^{\prime}\right\rangle$, has the same orbits on $\Omega$ as $\left\langle t_{i}\right\rangle$, respectively $\left\langle x_{i}\right\rangle$, and for which the product $t_{i}^{\prime} x_{i}^{\prime}$ has order coprime to $p$. By Theorem 1.2 the distance between such elements $t_{i}$ and $t_{i}^{\prime}$ in the relevant coprimality graph of $\operatorname{Sym}\left(\operatorname{supp}\left(t_{i}\right)\right)$ is at most 2, and using Corollary 3.8 if necessary there is a path of length exactly 2. Also, for the disjoint pairs $\left(t_{j}, x_{j}\right)$, Proposition 3.6 implies the existence of a $p$-cycle $y_{j}$ adjacent to both in the relevant coprimality graph of $\operatorname{Sym}\left(\operatorname{supp}\left(t_{j}\right) \cup \operatorname{supp}\left(x_{j}\right)\right)$. Let $t^{\prime}=t_{1}^{\prime} \ldots t_{l}^{\prime} x_{l+1} \ldots x_{v}$. Since the cycles $x_{l+1}, \ldots, x_{v}$ are disjoint from $t$, this element has cycle type $p^{r}$, and so lies in $X$. Also, by the above observations, $d\left(t, t^{\prime}\right) \leq 2$ in $\mathcal{C}_{p^{\prime}}(G, X)$. Now let $x^{\prime}=x_{1}^{\prime} \ldots x_{l}^{\prime} x_{l+1}^{-1} \ldots x_{v}^{-1}$. This is adjacent to $t^{\prime}$ in $\mathcal{C}_{p^{\prime}}(G, X)$, and now using Theorem 1.2 and Corollary 3.8 if necessary we see that $d\left(x^{\prime}, x\right) \leq 2$. We therefore have a path of length at most 5 between $t$ and $x$. Thus $\operatorname{Diam}\left(\mathcal{C}_{p^{\prime}}(G, X)\right) \leq 5$.

Now let $X$ be the $G$-conjugacy class of elements with cycle type $p^{r}$, where $p \geq 5$. Write $r=2 m$ or $r=2 m+1$ if $r$ is even or odd respectively. For $z \in X$, after fixing a left-to-right ordering of the disjoint cycles of $z$, denote by $\Lambda_{z}$ the support of the first $m$ cycles, and by $\Phi_{z}$ the support of the remaining cycles. 
Lemma 3.16. Let $t, x \in X$. Then there exists an element $y \in X$ such that $\Lambda_{t} \cup \Lambda_{y}$ is disjoint from $\Phi_{t} \cup \Phi_{y}$, and $d(x, y)=1$ in $\mathcal{C}_{p^{\prime}}(G, X)$.

Proof. Let

$$
x=\left(x_{1,1}, \ldots, x_{1, p}\right)\left(x_{2,1}, \ldots, x_{2, p}\right) \ldots\left(x_{r, 1}, \ldots, x_{r, p}\right) .
$$

Then set

$$
y=x^{-1}=\left(x_{1, p}, \ldots, x_{1,1}\right)\left(x_{2, p}, \ldots, x_{2,1}\right) \ldots\left(x_{r, p}, \ldots, x_{r, 1}\right) .
$$

Fix an ordering of the cycles of $t$. Choose $m$ cycles of $y$ such that the intersection of the support of these cycles with $\Lambda=\Lambda_{t}$ is as large as possible. Without loss of generality we assume $y$ is labelled so that these cycles are $y_{1}, \ldots, y_{m}$.

We wish to rearrange the elements of $\operatorname{supp}(y)$ and the cycles of $y$ to get an element $y^{\prime} \in X$, such that $d\left(x, y^{\prime}\right)=1$, and the support of the first $m$ cycles of $y^{\prime}$ contains only $\Lambda$ and elements of fix $(t)$. Let

$$
\Psi=\operatorname{supp}(y) \cap \operatorname{fix}(t),
$$

and let $l=|\Lambda \cap \operatorname{supp}(y)|$. Reading $y$ from left to right, collect the first $m p-l$ elements which lie in $\Psi$ into a set $\Sigma^{\prime}$. We now define

$$
\Sigma=(\Lambda \cap \operatorname{supp}(y)) \cup \Sigma^{\prime} .
$$

We aim to have the support of the first $m$ cycles of $y^{\prime}$ equal to $\Sigma$, which will ensure $y^{\prime}$ satisfies the first requirement of the lemma.

(3.3) For each cycle $y_{1}, \ldots, y_{m}$ at least one element lies in $\Sigma$.

For a contradiction suppose some cycle $y_{i}$ does not contain an element of $\Sigma$. If an element of $\Lambda$ lies in any cycle $y_{m+1}, \ldots, y_{r}$, then swapping this cycle with $y_{i}$ contradicts our choice of the first $m$ cycles of $y$. The only other possibility is that

$$
\operatorname{supp}\left(y_{i}\right) \cup \operatorname{supp}\left(y_{m+1}\right) \cup \ldots \cup \operatorname{supp}\left(y_{r}\right) \subseteq \operatorname{supp}\left(t_{m+1}\right) \cup \ldots \cup \operatorname{supp}\left(t_{r}\right)
$$

which is a contradiction since the cycles of $y$ are disjoint. This proves (3.3).

Suppose there is some cycle $y_{j}$ of $y$, where $j>m$, and $\operatorname{supp}\left(y_{j}\right) \subseteq \Sigma$. We then choose a cycle $y_{i}, i \leq m$, where $\operatorname{supp}\left(y_{i}\right) \nsubseteq \Sigma$ (such a cycle certainly exists, since $|\Sigma|=m p$ ), and swap the positions of these cycles in $y$. We do similarly for all such cycles. Therefore by (3.3), after the reordering of cycles, and possible cyclic reordering within individual cycles, we may assume without loss that, reading from left to right, the first elements of the first $m$ cycles of $y$ lie in $\Sigma$, while the last elements of the remaining cycles of $y$ lie outside $\Sigma$. We now fix this expression for $y$, so we do not allow any further reordering of cycles, or of elements within cycles.

Set $y=y^{(1)}$. Reading $y^{(1)}$ from left to right, take the first element of $\Lambda_{y^{(1)}}$ which does not lie in $\Sigma$, and the first element of $\Phi_{y^{(1)}}$ which does lie in $\Sigma$, and 
swap these to get an element $y^{(2)}$. Now, reading $y^{(2)}$ from left to right, take the first element of $\Lambda_{y^{(2)}}$ which does not lie in $\Sigma$, and the first element of $\Phi_{y^{(2)}}$ which does lie in $\Sigma$, and swap these to get an element $y^{(3)}$, and so on. Continuing in this fashion, we will eventually get an element $y^{\prime}=y^{(q)}$ where $\Lambda_{y^{(q)}}=\Sigma$.

We must now show that $d\left(x, y^{\prime}\right)=1$. We claim that any cycle of $x y^{\prime}$ has length at most 3. Suppose $\alpha_{k}$ is an element of some cycle of $x y^{\prime}$ which also lies in the cycle $\alpha$ of $x$. Let $x$ be labelled so that it acts in the standard way on the indices $\{1, \ldots, k\}$, so $\alpha_{i} x=\alpha_{i+1}$ (modulo $p$ ). Since $y=x^{-1}$, we have $\alpha_{i} y=\alpha_{i-1}$.

Suppose that $\alpha_{k+1}$ has been swapped (so $k \neq p-1$ ) with an element $\beta_{s+1}$ of some cycle $\beta$ of $x$. Note our expression for $y$ ensures that $s \neq 0$. Now $\beta_{s}$ may also have been swapped, but since we read from left to right and $\beta_{s}$ comes after $\beta_{s+1}$ in $y$, this swap must have been with some element to the right of $\alpha_{k+1}$ in $y$. If this element is $\alpha_{k}$, then clearly $\alpha_{k}$ is fixed by $x y^{\prime}$. So suppose it is a different element $\gamma_{u}$ from a cycle $\gamma$, another of the first $m$ cycles of $y$ (note the possibility that $\gamma=\alpha$ ). By our expression for $y, u \neq p$. But now $\gamma_{u+1}$ cannot have been swapped, since if it were it would have to be with an element between $\beta_{s}$ and $\beta_{s+1}$, a contradiction. Similarly, $\alpha_{k}$ also cannot be swapped. We thus have

$$
y^{\prime}=\ldots\left(\ldots, \beta_{s+1}, \alpha_{k}, \ldots\right) \ldots\left(\ldots, \gamma_{u+1}, \beta_{s}, \ldots\right) \ldots\left(\ldots, \alpha_{k+1}, \gamma_{u}, \ldots\right) \ldots,
$$

(where only the relevant cycles of $y^{\prime}$ are shown). So $\alpha_{k}$ is contained in a 3-cycle, namely $\left(\alpha_{k}, \gamma_{u}, \beta_{s}\right)$.

It is of course possible that not all elements in the above description have been swapped. However, by similar reasoning to that above, the effect of any non-swapping either gives another 3-cycle or decreases the length of the cycle containing $\alpha_{k}$. Thus the length of any cycle in $z y^{\prime}$ is at most 3. Since $p>3$ this shows that $d\left(x, y^{\prime}\right)=1$, and completes the proof of the lemma.

Finally, with Lemma 3.16 to hand, we may prove Theorem 1.4, which, again for the reader's convenience, we restate.

Theorem 3.17. Suppose that $G=\operatorname{Sym}(n)$ and $X$ is the $G$-conjugacy class of elements of cycle type $p^{r}$, where $p \geq 5$ is prime. Let $k$ be the least non-negative integer such that $r / 2^{k} \leq\lfloor\sqrt{p}\rfloor$. Then $\operatorname{Diam}\left(\mathcal{C}_{p^{\prime}}(G, X)\right) \leq 5+k$.

Proof. When $r \leq\lfloor\sqrt{p}\rfloor$ we have $k=0$, and the result holds by Theorem 1.3. So suppose $r>\lfloor\sqrt{p}\rfloor$, and let $t, x \in X$. Write $r=2 m$ or $r=2 m+1$ if $r$ is even or odd respectively. By Lemma 3.16 there exists an element $y$ such that $d(x, y)=1$, and we can write $t=t_{1} t_{2}, y=y_{1} y_{2}$, where $t_{1}, y_{1}$ have cycle type $p^{m}, t_{2}, y_{2}$ have cycle type $p^{m}$ or $p^{m+1}$, and the support $\Lambda$ of $t_{1}$ and $y_{1}$ is disjoint from the support $\Phi$ of $t_{2}$ and $y_{2}$. If $r=2 m$, then since $r / 2^{k} \leq\lfloor\sqrt{p}\rfloor$, we have $m / 2^{k-1} \leq\lfloor\sqrt{p}\rfloor$. Furthermore, if $r=2 m+1$, it is also the case that $(m+1) / 2^{k-1} \leq\lfloor\sqrt{p}\rfloor$. Indeed, since $(2 m+1) / 2^{k} \leq\lfloor\sqrt{p}\rfloor$ we have $2 m+1 \leq 2^{k}\lfloor\sqrt{p}\rfloor$. But since $2^{k}\lfloor\sqrt{p}\rfloor$ is even, this implies that $2 m+2 \leq 2^{k}\lfloor\sqrt{p}\rfloor$, whence $(m+1) / 2^{k-1} \leq\lfloor\sqrt{p}\rfloor$. Therefore, by induction there exist paths of length at most $5+(k-1)$ in the 
relevant coprimality graphs of $\operatorname{Sym}(\Lambda)$ and $\operatorname{Sym}(\Phi)$. Since $\Lambda$ and $\Phi$ are disjoint, the products of elements from these paths are elements of $X$. We therefore have a path of length at most $5+(k-1)+1$ from $t$ to $x$, as required.

\section{Connectedness of $\mathcal{C}_{\pi}(G, X)$}

As promised, in this short section we prove Theorem 1.1, which we restate.

Theorem 4.1. Suppose that $G=\operatorname{Sym}(n)$ and that $x$ is an element of order $p$, $p$ a prime. Let $X$ be the $G$-conjugacy class of $x$. Then $\mathcal{C}_{p^{\prime}}(G, X)$ is connected unless $n=4$ and $x$ has cycle type $2^{2}$.

Proof. Let $t \in X$ be such that $t=t_{1} t_{2} \cdots t_{r}$, where $t_{i}$ is the $p$-cycle

$$
((i-1) p+1,(i-1) p+2, \ldots, i p)
$$

for $i=1, \ldots, r$. Also $\mathcal{O}_{1}, \mathcal{O}_{2}, \ldots, \mathcal{O}_{r}$ will denote the orbits of $\langle t\rangle$ on $\Omega$ of length p. So

$$
\mathcal{O}_{i}=\{(i-1) p+1,(i-1) p+2, \ldots, i p\} .
$$

Set $\Phi=\bigcup_{i=1}^{r} \mathcal{O}_{i}$ and $\Lambda=\Omega \backslash \Phi$. Let $Y$ denote the connected component of $t$ in $\mathcal{C}_{p^{\prime}}(G, X)$, and set $K=\operatorname{Stab}_{G}(Y)$. By Theorem 2.2 the local fusion graph $\mathcal{F}(G, X)$ is connected for $n \geq 5$, and by checking the cases $n<5$ we see the theorem holds for $p=2$. So we may suppose $p$ is odd. Let $x \in X$. If $\langle x\rangle$ has the same orbits on $\Omega$ as $\langle t\rangle$, then $x \in Y$ by Theorem 1.2. Let $H$ denote the stabilizer in $G$ of the partition of $\Omega$ given by the orbits of $\langle t\rangle$. We note that $H=J \times L$, where $J \cong \operatorname{Sym}(p)$ < $\operatorname{Sym}(r)$ (with the base group being $\left.\operatorname{Sym}\left(\mathcal{O}_{1}\right) \times \cdots \times \operatorname{Sym}\left(\mathcal{O}_{r}\right)\right)$ and $L=\operatorname{Sym}(\Lambda)$. Thus we have $H \leq K$. We next show that $\operatorname{Sym}(\Phi) \times \operatorname{Sym}(\Lambda) \leq K$. If $r=1$, then we have this immediately. So we may suppose $r \geq 2$. Let $y=y_{1} y_{2} \cdots y_{r}$ be the product of pairwise disjoint cycles $y_{i}$, where

$$
\begin{aligned}
& y_{1}=(2,1, p+1, p+2, \ldots, 2 p-2) \\
& y_{2}=(2 p-1,2 p, 3,4, \ldots, p)
\end{aligned}
$$

and $y_{j}=t_{j}^{-1}$ for $j \geq 3$. So $y \in X$ and

$$
\begin{aligned}
t y=(1)(2,4,6, \ldots, p-1,2 p-1,3,5, \ldots \\
\\
\quad \ldots, p, p+1, p+3, p+5, \ldots, 2 p-2,2 p, p+2, p+4, \ldots, 2 p-3)
\end{aligned}
$$

which has order $2 p-1$. As a result $t$ and $y$ are adjacent in $\mathcal{C}_{p^{\prime}}(G, X)$ and we infer that $y \in K$. Since $y \in \operatorname{Sym}(\Phi) \backslash J$ and $J$ is a maximal subgroup of $\operatorname{Sym}(\Phi)$, we deduce that $\operatorname{Sym}(\Phi) \times \operatorname{Sym}(\Lambda) \leq K$.

If $\Lambda=\emptyset$, then we obtain $K=G$ whence $\mathcal{C}_{p^{\prime}}(G, X)$ is connected. So we now suppose $\Lambda \neq \emptyset$ and select $\alpha \in \Lambda$. Consider $z=z_{1} z_{2} \cdots z_{r} \in X$, where

$$
z_{1}=(2,3, \ldots, p, \alpha)
$$


and $z_{j}=t_{j}^{-1}$ for $j \geq 2$. Then

$$
t z=(1,3,5, \ldots, p-2, p)(2,4, \ldots, p-1, \alpha)
$$

which has order $(p-1) / 2$. So $t$ and $z$ are adjacent and thus $z \in K$. But $z \notin \operatorname{Sym}(\Phi) \times \operatorname{Sym}(\Lambda)$, which is a maximal subgroup of $G$. Therefore $K=G$ and $\mathcal{C}_{p^{\prime}}(G, X)$ is connected, so proving the theorem.

\section{References}

[1] M. Aschbacher: Finite group theory, Second edition. Cambridge Studies in Advanced Mathematics, 10. Cambridge University Press, Cambridge, 2000.

[2] J. Ballantyne, N. Greer, P. Rowley: Local Fusion Graphs for Symmetric Groups, J. Group Theory, to appear.

[3] C. Bates, D. Bundy, S. Perkins, P. Rowley: Commuting Involution Graphs for Symmetric Groups, J. Algebra 266 (2003), 133-153.

[4] C. Bates, D. Bundy, S. Perkins, P. Rowley: Commuting Involution Graphs for Finite Coxeter Groups, J. Group Theory 6 (4) (2003), 461-476.

[5] C. Bates, D. Bundy, S. Perkins, P. Rowley: Commuting involution graphs in special linear groups, Comm. Algebra 32 (2004), no. 11, 4179-4196.

[6] C. Bates, D. Bundy, S. Perkins, P. Rowley: Commuting Involution Graphs for Sporadic Groups, J. Algebra 316(2007), 849 - 868.

[7] C. Bates, D. Bundy, S. Perkins, P. Rowley. A Note on Commuting Graphs for Symmetric Groups, Elec. J. Combinatorics 16(2009), R6.

[8] W. Bosma, J. Cannon, C. Playoust: The Magma algebra system. I. The user language, J. Symbolic Comput., 24(3-4) (1997), 235-265.

[9] A. Devillers, M. Giudici: Involution graphs where the product of two adjacent vertices has order three, J. Aust. Math. Soc. 85 (2008), no. 3, 305-322.

[10] B. Sagan: The symmetric group. Representations, combinatorial algorithms, and symmetric functions, Second edition. Graduate Texts in Mathematics, 203. Springer-Verlag, New York (2001). xvi+238 pp. 